\title{
VARIATIONAL APPROXIMATION FOR DETECTING POINT-LIKE TARGET PROBLEMS *
}

\author{
Gilles AuberT ${ }^{1}$ And Daniele Graziani ${ }^{2}$
}

\begin{abstract}
The aim of this paper is to provide a rigorous variational formulation for the detection of points in 2-d biological images. To this purpose we introduce a new functional whose minimizers give the points we want to detect. Then we define an approximating sequence of functionals for which we prove the $\Gamma$-convergence to the initial one.
\end{abstract}

Mathematics Subject Classification. 49J45, 49Q20.

Received June 24, 2009. Revised November 16, 2009.

Published online August 6, 2010.

\section{INTRODUCTION}

The issue of detecting fine structures, like points or curves in two or three dimensional biological images, is a crucial task in image processing. In particular a point may represent a viral particle whose visibility is compromised by the presence of other structures like cell membranes or some noise. Therefore one of the main goals is to detect spots that biologists wish to count. This task is hard because of the presence of other singular structures.

In some biological images the image intensity is a function that takes the value 1 on points or other structures like sets with Hausdorff dimension $0 \leq \alpha<1$, and it is close to zero outside. In image processing these concentration sets are called discontinuities without jump, meaning that there is no jump across the set and therefore the gradient of the image is zero.

In the literature there are few variational methods dealing with this problem. In this direction an interesting approach has been proposed in [3]. In that paper the authors consider this kind of pathology as a $k$-codimension object, meaning that they should be regarded as a singularity of a map $U: \mathbb{R}^{k+m} \rightarrow \mathbb{R}^{k}$, with $k \geq 2$ and $m \geq 0$ (see [6] for a complete survey on this subject). In particular the detecting point case corresponds to the case $k=2$ and $m=0$.

This point of view makes possible a variational approach based on the theory of Ginzburg-Landau systems. In their work the isolated points in 2-D images are regarded as the topological singularities of a map $U: \mathbb{R}^{2} \rightarrow \mathbb{S}^{1}$, where $\mathbb{S}^{1}$ is the unit sphere of $\mathbb{R}^{2}$. Starting from the initial image $I: \Omega \subset \mathbb{R}^{2} \rightarrow \mathbb{R}$, this strategy makes crucial

Keywords and phrases. Points detection, biological images, divergence-measure fields, $p$-capacity, $\Gamma$-convergence.

* The research of Daniele Graziani was supported by ANR under the research project "Detectfine" (Laboratory I3S, Université de Nice Sophia Antipolis).

${ }^{1}$ Laboratoire J.A. Dieudonné, Université de Nice Sophia Antipolis, Parc Valrose, 06108 Nice Cedex 2, France. gaubert@unice.fr

2 Ariana CNRS/INRIA/UNSA Sophia Antipolis, Inria, 2004 route des Lucioles, BP 93, 06902 Sophia Antipolis Cedex, France.

Daniele.Graziani@sophia.inria.fr 
the construction of an initial vector field $U_{0}: \mathbb{R}^{2} \rightarrow \mathbb{S}^{1}$ with a topological singularity of degree one. Nevertheless how to obtain such a vector field in a rigorous way, seems to be still unclear.

Therefore one of the main purposes here is to find a most natural variational framework, in which a rigorous definition of discontinuity without jump can be given. In our model the image $I$ is a Radon measure. It is crucial for detecting points that this Radon measure would be able of charging points. The preliminary step is finding a space whose elements are able of producing this kind of measures. This space is given by $\mathcal{D} \mathcal{M}^{p}(\Omega)$ : the space of $L^{p}$-vector fields whose distributional divergence is a Radon measure, with $1<p<2$ (see Sect. 2 below). The restriction on $p$ is due to the fact that when $p \geq 2$ the distributional divergence $\operatorname{Div} U$ of $U$ cannot be a measure concentrated on points (see Sect. 3.1 below). Then we have to construct, from the original image $I$, a data vector $U_{0} \in \mathcal{D} \mathcal{M}^{p}(\Omega)$. Clearly there are, at least in principle, many ways to do this. The one we propose here seems to be the most natural. We consider the classical elliptic problem with measure data $I$ :

$$
\begin{cases}-\Delta u_{0}=I & \text { on } \Omega \\ u_{0}=0 & \text { on } \partial \Omega\end{cases}
$$

Then by setting $U_{0}=-\nabla u_{0}$ we have $U_{0} \in \mathcal{D} \mathcal{M}^{p}(\Omega)$ with $\operatorname{Div} U_{0}=I$. However the support of the measure $\operatorname{Div} U_{0}$ is too large and could contain several structures like curves or fractals, while the singularities, we are interested in, are contained in the atomic part of the measure $\operatorname{Div} U_{0}$ and therefore we have to isolate them. To do this the notion of $p$-capacity of a set plays a key role. Indeed when $p<2$ the $p$-capacity of a point in $\Omega$ is zero and one can say, in this sense, that it is a discontinuity without jump. Besides every Radon measure can be decomposed in two mutually singular measures: the first one is absolutely continuous with respect to the $p$-capacity and the second one singular with respect to the $p$-capacity, that is a measure concentrated on sets with zero $p$-capacity (see [14]).

As it is known in dimension two, sets with zero $p$-capacity, and hence discontinuities without jump, can be isolated points, countable set of points or fractals with Hausdorff dimension $0 \leq \alpha<1$ (see Sect. 2.3 for the definition of $p$-capacity and related properties).

Our goal here is keeping nothing else but points in the image. The achievement of such a purpose makes necessary the minimization of a suitable energy that must remove all the discontinuities which are not discontinuities without jump, and remove all the discontinuities without jump which are not isolated points.

From one hand we have to force the concentration set of the divergence measure of $U$ to contain only the points we want to detect, and on the other hand we have to regularise the initial data $U_{0}$ outside points of singularities. To this end we introduce the auxiliary space $\operatorname{SD}_{\mathcal{M}}^{p}(\Omega)$ of vector fields belonging to $\mathcal{D} \mathcal{M}^{p}(\Omega)$ whose divergence measure has no absolutely continuous part with respect to the $p$-capacity. Then, by taking into account that the initial vector field is a gradient of a Sobolev function, our goal is to minimize the following energy:

$$
\mathcal{F}(u)=\int_{\Omega}|\Delta u|^{2} \mathrm{~d} x+\lambda \int_{\Omega}\left|\nabla u-U_{0}\right|^{p} \mathrm{~d} x+\mu \mathcal{H}^{0}\left(\operatorname{supp}\left(\operatorname{div}^{s} \nabla u\right)\right),
$$

where $u \in W_{0}^{1, p}(\Omega)$ with $\nabla u \in S \mathcal{D} \mathcal{M}^{p}(\Omega), 1<p<2$ and $\lambda, \mu$ are positive weights. The gradient of a minimizer of the energy $\mathcal{F}$ is the vector field we are looking for, that is a vector field whose divergence measure can be decomposed in an absolutely continuous term (with respect to the Lebesgue's measure) plus an atomic measure concentrated on the points we want to isolate in the image.

Even if a pointwise characterization of discontinuity without jump sets is not available, thanks to our definition the singular set of points can be linked to the vector field $\nabla u$, in spirit of the classical SBV formulation of the Mumford-Shah's functional (we refer to [1] for a complete survey on the Mumford Shah's functional).

For computational purposes, the next task is to provide an approximation in the sense of $\Gamma$-convergence introduced in $[16,17]$. Our approach is close in the spirit to the one used to approximate the Mumford Shah's functional by a family of depending curvature functionals as in [9]. Indeed, as in their work (see also [8]), 
we replace the atomic measure $\mathcal{H}^{0}$ by the term

$$
G_{\varepsilon}(D)=\frac{1}{4 \pi} \int_{\partial D}\left(\frac{1}{\varepsilon}+\varepsilon \kappa^{2}\right) \mathrm{d} \mathcal{H}^{1}
$$

where $D$ is a regular set, $\kappa$ is the curvature of its boundary, and the constant $\frac{1}{4 \pi}$ is a normalization factor. Roughly speaking the minima of these functionals are achieved on the union of balls of small radius, so that when $\varepsilon \rightarrow 0$ the sequence $G_{\varepsilon}$ shrinks to the atomic measure $\mathcal{H}^{0}$.

This leads to an intermediate approximation given by

$$
F_{\varepsilon}(u, D)=\int_{\Omega}\left(1-\chi_{D}\right)|\Delta u|^{2} \mathrm{~d} x+\lambda \int_{\Omega}\left|\nabla u-U_{0}\right|^{p} \mathrm{~d} x+\mu \frac{1}{4 \pi} \int_{\partial D}\left(\frac{1}{\varepsilon}+\varepsilon \kappa^{2}\right) \mathrm{d} \mathcal{H}^{1}
$$

This strategy permits to work with the perimeter measure $\mathcal{H}^{1}\lfloor\partial D$, that can be approximated, according to the Modica-Mortola's approach (see [21,22]), by the measure:

$$
\mu_{\varepsilon}(w, \nabla w)=\left(\varepsilon|\nabla w|^{2}+\frac{W(w)}{\varepsilon}\right) \mathrm{d} x
$$

where $W(w)=w^{2}(1-w)^{2}$ is a double well function.

Besides by using Sard's Theorem and coarea formula (see also [4] for a similar approach) one can formally replace the integral on $\partial D$ by an integral computed over the level sets of $w$, whose curvature $\kappa$ becomes div $\frac{\nabla w}{|\nabla w|}$ and the integral is computed over the level sets of $w$. So that one can formally write the complete approximating sequence:

$$
\begin{aligned}
\mathcal{G}_{\varepsilon}(u, w)= & \int_{\Omega} w^{2}|\Delta u|^{2} \mathrm{~d} x+\mu \frac{1}{8 \pi C} \int_{\Omega \backslash\{|\nabla w=0|\}}\left(\frac{1}{\beta_{\varepsilon}}+\beta_{\varepsilon}\left(\operatorname{div}\left(\frac{\nabla w}{|\nabla w|}\right)\right)^{2}\right)\left(\varepsilon|\nabla w|^{2}+\frac{1}{\varepsilon} W(w)\right) \mathrm{d} x \\
& +\lambda \int_{\Omega}\left|\nabla u-U_{0}\right|^{p} \mathrm{~d} x+\frac{1}{\gamma_{\varepsilon}} \int_{\Omega}(1-w)^{2} \mathrm{~d} x,
\end{aligned}
$$

where, as usual, $C=\int_{0}^{1} \sqrt{W(t)} \mathrm{d} t, \beta_{\varepsilon}$ and $\gamma_{\varepsilon}$ are infinitesimal as $\varepsilon \rightarrow 0$. The last integral is a penalization term that forces $w$ to tend to 1 as $\varepsilon \rightarrow 0$.

Thus the main goal of this work is to show that the family of energies $\mathcal{G}_{\varepsilon} \Gamma$-converges to the functional $\mathcal{F}$ when the parameters are related in a suitable way.

As in [9] we deal with a suitable convergence of functions involving the Hausdorff convergence of a sub-level sets. This strategy requires a careful statement of the $\Gamma$-convergence definitions and results, in order to have that sequences asymptotically minimizing $\mathcal{G}_{\varepsilon}$ converge to a minimum of $\mathcal{F}$.

Although this approach is inspired by some ideas contained in [8,9], we point out that in our case the regularization term involves a second order differential operator, due to the fact that our goal is to detect points and not segment curves. This deep difference requires a non trivial adaptation of the arguments used in those papers.

The paper is organized as follows. Section 2 is devoted to notations, preliminary definitions and results. In Section 3 we illustrate the new variational model and we present the functional we deal with. Sections 4 and 5 are devoted to the $\Gamma$-convergence results. Finally in the last section we conclude the paper by comparing this approach with the celebrated conjecture by De Giorgi, concerning the approximation of the curvature depending functionals.

We do not give here experimental result illustrating our approach. We refer the reader for that to [19]. 


\section{Definition AND MAIN PROpERTIES}

\subsection{Notation}

In all the paper $\Omega \subset \mathbb{R}^{2}$ is an open bounded set with Lipschitz boundary. The Euclidean norm will be denoted by $|\cdot|$, while the symbol $\|\cdot\|$ indicates the norm of some functional spaces. The brackets $\langle$,$\rangle denotes$ the duality product in some distributional spaces. $\mathcal{L}^{d}$ or $d x$ is the $d$-dimensional Lebesgue measure and $\mathcal{H}^{k}$ is the $k$-dimensional Hausdorff measure. $B_{\rho}\left(x_{0}\right)$ is the ball centered at $x_{0}$ with radius $\rho$. We say that a set $D \subset \Omega$ is a regular set if it can be written as $\{F<0\}$ with $F \in C_{0}^{\infty}(\Omega)$. In the following we will denote by $R(\Omega)$ the family of all regular sets in $\Omega$. Finally we will use the symbol $\rightarrow$ for denoting a weak convergence.

\subsection{Distributional divergence and classical spaces}

In this section we recall the definition of the distributional space $L^{p, q}(\operatorname{div} ; \Omega)$ and $\mathcal{D} \mathcal{M}^{p}(\Omega), 1 \leq p, q \leq+\infty$, $($ see $[2,11])$.

Definition 2.1. We say that $U \in L^{p, q}(\operatorname{div} ; \Omega)$ if $U \in L^{p}\left(\Omega ; \mathbb{R}^{2}\right)$ and if its distributional divergence $\operatorname{Div} U=$ $\operatorname{div} U \in L^{q}(\Omega)$. If $p=q$ the space $L^{p, q}(\operatorname{div} ; \Omega)$ will be denoted by $L^{p}(\operatorname{div} ; \Omega)$.

We say that a function $u \in W^{1, p}(\Omega)$ belongs to $W^{1, p, q}(\operatorname{div} ; \Omega)$ if $\nabla u \in L^{p, q}(\operatorname{div} ; \Omega)$. We say that a function $u \in W_{0}^{1, p}(\Omega)$ belongs to $W_{0}^{1, p, q}(\operatorname{div} ; \Omega)$ if $\nabla u \in L^{p, q}(\operatorname{div} ; \Omega)$.

Definition 2.2. For $U \in L^{p}\left(\Omega ; \mathbb{R}^{2}\right), 1 \leq p \leq+\infty$, set

$$
|\operatorname{Div} U|(\Omega):=\sup \left\{\langle U, \nabla \varphi\rangle: \varphi \in C_{0}^{\infty}(\Omega),|\varphi| \leq 1\right\} .
$$

We say that $U$ is an $L^{p}$-divergence measure field, i.e. $U \in \mathcal{D} \mathcal{M}^{p}(\Omega)$, if

$$
\|U\|_{\mathcal{D} \mathcal{M}^{p}(\Omega)}:=\|U\|_{L^{p}\left(\Omega ; \mathbb{R}^{2}\right)}+|\operatorname{Div} U|(\Omega)<+\infty .
$$

Let us recall the following classical result (see [12], Prop. 3.1).

Theorem 2.1. Let $\left\{U_{h}\right\}_{h} \subset \mathcal{D M}^{p}(\Omega)$ be such that

$$
U_{h} \rightarrow U \quad \text { in } L^{p}\left(\Omega ; \mathbb{R}^{2}\right) \text {, as } h \rightarrow+\infty \text { for } 1 \leq p<+\infty .
$$

Then

$$
\|U\|_{L^{p}\left(\Omega ; \mathbb{R}^{2}\right)} \leq \liminf _{h \rightarrow+\infty}\left\|U_{h}\right\|_{L^{p}\left(\Omega ; \mathbb{R}^{2}\right)}, \quad|\operatorname{Div} U|(\Omega) \leq \liminf _{h \rightarrow+\infty}\left|\operatorname{Div} U_{h}\right|(\Omega) .
$$

\section{3. $p$-Capacity}

The notion of $p$-capacity of sets will be crucial to find a convenient functional framework to deal with. If $K \subset \mathbb{R}^{2}$ is a compact set and $\chi_{K}$ denotes its characteristic function, we define:

$$
\operatorname{Cap}_{p}(K, \Omega)=\inf \left\{\int_{\Omega}|\nabla f|^{p} \mathrm{~d} x, f \in C_{0}^{\infty}(\Omega), f \geq \chi_{k}\right\} .
$$

If $U \subset \Omega$ is an open set and $K \subset U$ is a compact set, its $p$-capacity is given by

$$
\operatorname{Cap}_{p}(U, \Omega)=\sup _{K \subset U} \operatorname{Cap}_{p}(K, \Omega) .
$$

Finally if $A \subset U \subset \Omega$ with $A$ Borel set and $U$ open, then

$$
\operatorname{Cap}_{p}(A, \Omega)=\inf _{A \subset U \subset \Omega} \operatorname{Cap}_{p}(U, \Omega) .
$$


We recall the following result (see for instance [20], Thm. 2.27) that explains the relationship between $p$-capacity and Hausdorff measures. Such a result is crucial to have geometric information on null $p$-capacity sets.

Theorem 2.2. Assume $1<p<2$. If $\mathcal{H}^{2-p}(A)<\infty$ then $\operatorname{Cap}_{p}(A, \Omega)=0$.

Another useful tool to manage sets of zero $p$-capacity is provided by the following characterization.

Theorem 2.3. Let $E$ be a compact subset of $\Omega$. Then $\operatorname{Cap}_{p}(E, \Omega)=0$ if and only if there exists a sequence $\left\{\phi_{k}\right\}_{k} \subset C_{0}^{\infty}(\Omega)$, converging to 0 strongly in $W_{0}^{1, p}(\Omega)$, such that $0 \leq \phi_{k} \leq 1$ and $\phi_{k}=1$ on $E$ for every $k$.

For a general survey we refer the reader to $[18,20,25]$.

\section{The VARIATIONAL MODEL}

In this section we set the functional framework and the functional to be minimized.

Roughly speaking in biological images the image is a function that could be very high on points or other structures like sets with Hausdorff dimension $0 \leq \alpha<1$, and it is close to zero outside. From a mathematical point of view it seems to be much more appropriate to think of the image as a Radon measure, that is $I=\mu \in$ $\left(C_{0}(\Omega)\right)^{*}$. The next step is finding a space whose elements are able of producing this kind of discontinuities: the space $\mathcal{D M}^{p}(\Omega)$, with $1<p<2$. The restriction on $p$ is due to the fact that when $p \geq 2$ the distributional divergence of $U$ cannot be a measure concentrated on points. Set $p \geq 2$, according to the definition, we have

$$
\langle\operatorname{Div} U, \varphi\rangle=-\int_{\Omega} U \cdot \nabla \varphi \mathrm{d} x \quad \text { for all } \varphi \in C_{0}^{\infty}(\Omega)
$$

Since $p \geq 2$ this distribution is well-defined for any test $\varphi \in W_{0}^{1, p^{\prime}}(\Omega)$, where $p^{\prime} \leq 2$ is the dual exponent of $p$. In particular $\operatorname{Div} U$ belongs to the dual space $W^{-1, p}(\Omega)$ of the Sobolev space $W_{0}^{1, p^{\prime}}(\Omega)$. Then in this case, the distributional divergence of $U$ cannot be an atomic measure, since $\delta_{0} \notin W^{-1, p}(\Omega)$. To see this, one can consider as $\Omega$ the disk $B_{1}(0)$ and the function $\tilde{\varphi}(x)=\log (\log (1+|x|))-\log (\log (2))$. This function is in the space $W_{0}^{1, p^{\prime}}(\Omega)$ for every $p^{\prime} \leq 2$ and therefore it is an admissible test function, however it easy to check that $\left\langle\delta_{0}, \tilde{\varphi}\right\rangle=+\infty$.

When $1<p<2$ we have that $\operatorname{Div} U \in W^{-1, p}(\Omega)$, but in this case since $p<2$, we have $p^{\prime}>2$ and hence the function $\tilde{\varphi}$ is no longer an admissible test function. One can check that the distribution Div $U$ is an element of $\left(C_{0}(\Omega)\right)^{*}$ able of charging the points. Take for instance the map $U(x, y)=\left(\frac{x}{x^{2}+y^{2}}, \frac{y}{x^{2}+y^{2}}\right)$.

The next step is to transform the initial image $I$ as the divergence measure of a suitable vector field. We consider the elliptic problem with measure data $I$ :

$$
\begin{cases}-\Delta u=I & \text { on } \Omega \\ u=0 & \text { on } \partial \Omega .\end{cases}
$$

Classical results (see [24]) ensures the existence of a unique weak solution $u \in W_{0}^{1, p}(\Omega)$ with $p<2$. Then it easy to see that the distributional divergence of $-\nabla u$ is given by $I$. In particular by setting $U=-\nabla u$, we have $U \in \mathcal{D M}^{p}(\Omega)$. According to the Radon-Nikodym decomposition of the measure Div $U$ we have

$$
\operatorname{Div} U=\operatorname{div} U \mathrm{~d} x+\operatorname{div}^{s} U
$$

where $\operatorname{div} U \in L^{1}(\Omega)$ and $\operatorname{div}^{s} U$ is a singular measure with respect to $\mathcal{L}^{2}$. For our purpose the support of the singular measure $\operatorname{div}^{s} U$ is too large. In particular the measure $\operatorname{div}^{s} U$ could charge sets with Hausdorff dimension $0 \leq \alpha<2$. So that in order to isolate the singularities we are interested in, we need a further decomposition 
of the measure DivU. This can be done by using the capacitary decomposition of the Radon measure $\operatorname{div}^{s} U$. It is known (see [14]) that given a Radon measure $\mu$ the following decomposition holds

$$
\mu=\mu_{a}+\mu_{0}
$$

where the measure $\mu_{a}$ is absolutely continuous with respect to the $p$-capacity and $\mu_{0}$ is singular with respect to the $p$-capacity, that is concentrated on sets with zero $p$-capacity. Besides it is also known (see [14]) that every measure which is absolutely continuous with respect to the $p$-capacity can be characterized as an element of $L^{1}+W^{-1, p^{\prime}}$, leading to the finer decomposition:

$$
\mu=f-\operatorname{Div} G+\mu_{0},
$$

where $G \in L^{p^{\prime}}\left(\Omega ; \mathbb{R}^{2}\right)$ with $\frac{1}{p}+\frac{1}{p^{\prime}}=1$ and $f \in L^{1}(\Omega)$. DivU

By applying this decomposition to the measure $\operatorname{div}^{s} U$ we obtain the following decomposition of the measure

$$
\operatorname{Div} U=\operatorname{div} U+f-\operatorname{Div} G+\left(\operatorname{div}^{s} U\right)_{0},
$$

with $G \in L^{p^{\prime}}\left(\Omega ; \mathbb{R}^{2}\right), f \in L^{1}(\Omega), \operatorname{div} U \in L^{1}(\Omega)$ and $\left(\operatorname{div}^{s} U\right)_{0}$ is a measure concentrated on a set with zero p-capacity.

According to this decomposition and taking into account Theorem 2.3 we give the definition of discontinuity without/with jump.

Definition 3.1. We say that a point $x \in \Omega \subset \mathbb{R}^{2}$ is a point of discontinuity without jump of $U$ if $x \in$ $\operatorname{supp}\left(\operatorname{div}^{s} U\right)_{0}$.

Definition 3.2. We say that a point $x \in \Omega \subset \mathbb{R}^{2}$ is a point of discontinuity with jump of $U$ if $x \in \operatorname{supp}(f-\operatorname{Div} G)$.

Remark 3.1. The other singularities, where there is a jump, are contained in the term $f$ - Div $G$ of decomposition (3.4). Indeed the space $W^{-1, p^{\prime}}(\Omega)$ contains Hausdorff measures restricted to sub-manifolds of dimension greater than or equal to one, like for instance Hausdorff measures concentrated on regular closed curves, which are classical examples of discontinuities with jump (we refer to [25], Sect. 4.7, for a detailed discussion on the space $\left.W^{-1, p^{\prime}}(\Omega)\right)$. More precisely a contour of a regular set $D$ is the jump set of the characteristic function of $D$ and its $p$-capacity is strictly positive. This is of course in agreement with Theorem 2.3. Indeed if there were a sequence $\left\{\phi_{k}\right\}_{k} \subset C_{0}^{\infty}(\Omega)$, converging to 0 strongly in $W_{0}^{1, p}(\Omega)$, such that $0 \leq \phi_{k} \leq 1$ and $\phi_{k}=1$ on $\partial D$ for every $k$, it would be possible to define the sequence

$$
\tilde{\phi}_{k}= \begin{cases}\phi_{k} & \text { on } D \\ 1 & \text { on } \Omega \backslash D\end{cases}
$$

which converges, in the $W^{1, p}$-norm, to the $B V$-function $1-\chi_{D}$, which cannot be approximated by regular functions in this norm.

\subsection{The variational framework}

We shall introduce an energy whose minimizers will be vector fields whose divergence measure's singular part will be given by nothing else but points.

Each minimizer must be an $L^{p}$ vector field (with $p<2$ ) with the following properties:

(1) It must be close to the initial data $U_{0}$ which is, in general, an $L^{p}$-vector field $U_{0}$ with $1<p<2$.

(2) The absolutely continuous part with respect to the Lebesgue measure of $\operatorname{Div} U$ is an $L^{2}$-function.

(3) The support of the measure $\left(\operatorname{div}^{s} U\right)_{0}$ must be given by a set of points $P_{U}$ with $\mathcal{H}^{0}\left(P_{U}\right)<+\infty$. 
According to these considerations it is natural to introduce the space

$$
\operatorname{SD}_{\mathcal{M}}(\Omega):=\left\{U \in \mathcal{D} \mathcal{M}^{p}(\Omega), \quad f-\operatorname{Div} G=0\right\},
$$

where $f$ and $G$ are given as in (3.3). So that, as a consequence, decomposition (3.4) yields for any $U \in S \mathcal{D} \mathcal{M}^{p}(\Omega)$

$$
\operatorname{Div} U=\operatorname{div} U+\left(\operatorname{div}^{s} U\right)_{0}
$$

For our purposes the following result concerning the features of elements of the space $S \mathcal{D} \mathcal{M}^{p}(\Omega)$ will play a crucial role.

Proposition 3.1. Let $P \subset \Omega$ be a set of finite number of points. Let $u \in W_{0}^{1, p, 2}(\operatorname{div} ; \Omega \backslash P)$, with $1<p<2$. Then $\nabla u \in \operatorname{SD}_{\mathcal{M}} \mathcal{M}^{2}(\Omega)$, with $\left(\operatorname{div}^{s} \nabla u\right)_{0}=P$.

Proof. We set $P=\left\{x_{1}, \ldots, x_{n}\right\}$. Let $\rho(h) \rightarrow 0$ as $h \rightarrow+\infty$ be such that $B_{\rho_{h}}\left(x_{i}\right) \cap B_{\rho_{h}}\left(x_{j}\right)=\emptyset$ for $h$ large enough and $i \neq j$. We set $\Omega_{h}=\bigcup_{i=1}^{n} B_{\rho_{h}}\left(x_{i}\right)$ and we define the following sequence $\left\{U_{h}\right\} \subset L^{p}\left(\Omega ; \mathbb{R}^{2}\right)$.

$$
\begin{cases}U_{h}=\nabla u & \text { on } \Omega \backslash \Omega_{h} \\ 0 & \text { on } \Omega_{h} .\end{cases}
$$

Since $\Delta u \in L^{2}(\Omega \backslash P)$, by standard elliptic regularity we deduce that $u \in W_{\text {loc }}^{2, p}(\Omega \backslash P)$. In particular, we infer that $u \in W^{2, p}\left(\Omega \backslash \Omega_{h}\right)$. For every $i=1, \ldots, n$ and $h$ small enough we can find an open set $A_{i}$ such that $B_{\rho_{h}}\left(x_{i}\right) \subset A_{i} \subset \Omega \backslash \bigcup_{j \neq i} B_{\rho_{h}}\left(x_{j}\right)$ and $A_{i}$ does not depend on $h$. Let $\theta_{i}$ be a cutoff function associated to $A_{i}$ such that

$$
\begin{cases}\theta_{i}=1 & \text { on } B_{\rho_{h}}\left(x_{i}\right) \text { for any } i=1, \ldots, n, \\ 0 \leq \theta_{i} \leq 1 & \text { for any } i=1, \ldots, n, \\ \theta_{i}=0 & \text { on } \Omega \backslash A_{i} \text { for any } i=1, \ldots, n, \\ \left\|\nabla \theta_{h}\right\|_{\infty} \leq \frac{M_{i}}{d\left(\partial A_{i}, \partial B_{\rho_{h}}\left(x_{i}\right)\right)} & \text { for any } i=1, \ldots, n,\end{cases}
$$

where $M_{i}$ is a suitable constant. Then, if $\varphi \in C_{0}^{1}(\Omega)$ with $|\varphi| \leq 1$, by applying Gauss-Green's formula we obtain:

$$
\begin{aligned}
\int_{\Omega} U_{h} \cdot \nabla \varphi \mathrm{d} x= & \int_{\Omega \backslash \Omega_{h}} \nabla u \cdot \nabla \varphi \mathrm{d} x=-\int_{\Omega \backslash \Omega_{h}} \Delta u \varphi \mathrm{d} x+\int_{\partial\left(\Omega \backslash \Omega_{h}\right)} \nabla u \cdot \nu \varphi \mathrm{d} \mathcal{H}^{1} \\
= & -\int_{\Omega \backslash \Omega_{h}} \Delta u \varphi \mathrm{d} x+\sum_{i=1}^{n} \int_{\partial\left(\Omega \backslash B_{\rho_{h}}\left(x_{i}\right)\right)} \nabla u \cdot \nu\left(\varphi-\theta_{i} \varphi\left(x_{i}\right)\right) \mathrm{d} \mathcal{H}^{1} \\
& +\sum_{i=1}^{n} \varphi\left(x_{i}\right) \int_{\partial\left(\Omega \backslash B_{\rho_{h}}\left(x_{i}\right)\right)} \theta_{i} \nabla u \cdot \nu \mathrm{d} \mathcal{H}^{1} \\
= & -\int_{\Omega \backslash \Omega_{h}}^{n} \Delta u \varphi \mathrm{d} x+\underbrace{\sum_{i=1}^{n} \int_{\partial B_{\rho_{h}}\left(x_{i}\right)} \nabla u \cdot \nu\left(\varphi-\varphi\left(x_{i}\right)\right) \mathrm{d} \mathcal{H}^{1}}_{=0} \\
& +\sum_{i=1}^{n} \varphi\left(x_{i}\right)\left\{\int_{A_{i} \backslash B_{\rho_{h}}\left(x_{i}\right)} \Delta u \theta_{i} \mathrm{~d} x+\int_{A_{i} \backslash B_{\rho_{h}}\left(x_{i}\right)} \nabla u \nabla \theta_{i} \mathrm{~d} x\right\}
\end{aligned}
$$

where in the last equality we have applied again the Gauss-Green's formula and the definition of $\theta_{i}$.

Now for every $i$ we have that $\left\{\partial B_{\rho_{h}}\left(x_{i}\right)\right\}$ converges in the Hausdorff metric to the singleton $\left\{x_{i}\right\}$. Then, since the support of the function $\psi=\varphi-\varphi\left(x_{i}\right)$ is contained in $\Omega \backslash\left\{x_{i}\right\}$, we have that $\operatorname{supp} \psi \cap \partial\left\{B_{h}\left(x_{i}\right)\right\}=\emptyset$ for $h$ large enough, by standard properties of the Hausdorff convergence. Therefore the third term in (3.9) 
is equal to 0 . Moreover for $h$ large enough we can find an open regular set $A$, that does not depend on $h$, such that $u \in W^{2, p}(\Omega \backslash A)$. Therefore, from (3.9) it follows that

$$
\begin{aligned}
\left|\operatorname{Div} U_{h}\right|(\Omega) \leq & \sup _{0 \leq \varphi \leq 1} \int_{\Omega \backslash \Omega_{h}}|\nabla u \cdot \nabla \varphi| \mathrm{d} x \leq(n+1) C_{1}(\Omega)\|\Delta u\|_{L^{2}(\Omega \backslash P)} \\
& +\|\nabla u\|_{L^{p}\left(\Omega ; \mathbb{R}^{2}\right)} \sum_{i=1}^{n} \frac{M_{i}}{d\left(\partial A_{i}, \partial B_{\rho_{h}}\left(x_{i}\right)\right)}:=C(n, \Omega),
\end{aligned}
$$

for $h$ large enough. Since $U_{h} \rightarrow \nabla u$ in $L^{p}\left(\Omega ; \mathbb{R}^{2}\right)$, by Theorem 2.1

$$
|\operatorname{Div} \nabla u|(\Omega) \leq \liminf _{h \rightarrow \infty}\left|\operatorname{Div} \nabla u_{h}\right| \leq C .
$$

Therefore $\nabla u \in \mathcal{D M}^{p}(\Omega)$. Finally we know that $u \in W^{1, p, 2}(\operatorname{div} ; \Omega \backslash P)$ and thus the support of the measure $\operatorname{div}^{s} \nabla u$ is given by the set $P$. Since $\operatorname{Cap}_{p}(P, \Omega)=0$, according to decomposition (3.4) the measure $f$ - DivG vanishes on sets with zero $p$-capacity, and we deduce $f-\operatorname{DivG}=0$, that is $\nabla u \in \operatorname{SDM}^{p}(\Omega)$, with $\left(\operatorname{div}^{s} \nabla u\right)_{0}=P$.

\subsection{The functional}

According to our purpose the natural energy to deal with is the following $F: S \mathcal{D} \mathcal{M}^{p}(\Omega) \rightarrow[0, \infty], 1<p<2$, given by

$$
F(U)=\int_{\Omega}|\operatorname{div} U|^{2} \mathrm{~d} x+\lambda \int_{\Omega}\left|U-U_{0}\right|^{p} \mathrm{~d} x+\mu \mathcal{H}^{0}\left(\operatorname{supp}\left(\operatorname{div}^{s} U\right)_{0}\right) .
$$

From now on we assume without loosing generality that the weights $\lambda$ and $\mu$ are equal to one.

We note that, if $\operatorname{Div} U_{0} \neq 0$ in $\mathcal{D}^{\prime}(\Omega)$, then $\inf F(U)>0$ on $S \mathcal{D} \mathcal{M}^{p}(\Omega)$. Indeed if we had $\inf _{S \mathcal{M} \mathcal{M}^{p}(\Omega)} F(U)=0$ then, it would be possible exhibiting a minimizing sequence $\left\{U_{n}\right\}$, such that $F\left(U_{n}\right) \rightarrow 0$. This would imply $U_{n} \rightarrow U_{0}$ in $L^{p}$ and $\operatorname{Div} U_{n} \rightarrow 0$ in $\mathcal{D}^{\prime}(\Omega)$. On the other hand, the $L^{p}$-distance between $U_{n}$ and $U_{0}$ can be arbitrary small only if $\operatorname{Div} U_{0}=0$ as well, because the constraint $\operatorname{Div} U=0$ is stable under $L^{p}$-convergence.

\section{T-CONVERGENCE: The Intermediate APPROXIMATION}

By analogy with the construction of $U_{0}$ we restrict ourselves to vector fields $U$ which are the gradient of a function $u \in W_{0}^{1, p}(\Omega)$.

Thus the functional $\mathcal{F}$ is finite on the class of functions whose support of the measure $\left(\operatorname{div}^{s} \nabla u\right)_{0}$ is given by a finite set. Consequently it is convenient to introduce the following spaces:

$$
\Delta \mathcal{M}^{p}(\Omega):=\left\{u \in W_{0}^{1, p}(\Omega), \nabla u \in \operatorname{SD}^{p}(\Omega)\right\}
$$

and

$$
\Delta \mathcal{A} \mathcal{M}^{p, 2}(\Omega)=\left\{u \in \Delta \mathcal{M}^{p}(\Omega): \Delta u \in L^{2}(\Omega), \operatorname{supp}\left(\operatorname{div}^{s} \nabla u\right)_{0}=P_{\nabla u} \text { with } \mathcal{H}^{0}\left(P_{\nabla u}\right)<+\infty\right\} .
$$

So that the target-limit energy $\mathcal{F}: \Delta \mathcal{A M}^{p, 2}(\Omega) \rightarrow(0, \infty)$ is given by

$$
\mathcal{F}(u)=\int_{\Omega}|\Delta u|^{2} \mathrm{~d} x+\int_{\Omega}\left|\nabla u-U_{0}\right|^{p} \mathrm{~d} x+\mathcal{H}^{0}\left(P_{\nabla u}\right) .
$$

In spirit of [9] we introduce an intermediate variational approximation of the functional $\mathcal{F}$. We define a sequence of functionals where the counting measure $\mathcal{H}^{0}\left(P_{\nabla u}\right)$ is replaced by a functional defined on regular sets $D$ 
and which involves the curvature of the boundary $\partial D$. The approximating sequence is given by:

$$
F_{\varepsilon}(u, D)=\int_{\Omega}\left(1-\chi_{D}\right)|\Delta u|^{2} \mathrm{~d} x+\int_{\Omega}\left|\nabla u-U_{0}\right|^{p} \mathrm{~d} x+\frac{1}{4 \pi} \int_{\partial D}\left(\frac{1}{\varepsilon}+\varepsilon \kappa^{2}\right) \mathrm{d} \mathcal{H}^{1}
$$

where $u \in W_{0}^{1, p, 2}(\operatorname{div} ; \Omega), D$ is a regular set, and $\kappa$ denotes the curvature of its boundary.

In order to guarantee that the measure of the sets $D$ is small we define a new functional still denoted by $F_{\varepsilon}(u, D)$ given by

$$
F_{\varepsilon}(u, D)=\int_{\Omega}\left(1-\chi_{D}\right)|\Delta u|^{2} \mathrm{~d} x+\int_{\Omega}\left|\nabla u-U_{0}\right|^{p} \mathrm{~d} x+\frac{1}{4 \pi} \int_{\partial D}\left(\frac{1}{\varepsilon}+\varepsilon \kappa^{2}\right) \mathrm{d} \mathcal{H}^{1}+\frac{1}{\varepsilon} \mathcal{L}^{2}(D) \quad \text { on } Y(\Omega)
$$

where $Y(\Omega)=\left\{(u, D), u \in W_{0}^{1, p, 2}(\operatorname{div} ; \Omega), D \in R(\Omega)\right\}$. We endow the set $Y(\Omega)$ with the following convergence.

Definition 4.1. We say that a sequence $\left\{\left(u_{h}, D_{h}\right)\right\}_{h} \subset Y(\Omega)$ H-converges to $u \in \Delta \mathcal{A} \mathcal{M}^{p, 2}(\Omega)$ if the following conditions hold:

(1) $\mathcal{L}^{2}\left(D_{h}\right) \rightarrow 0$

(2) $\left\{\partial D_{h}\right\}_{h} \rightarrow P \subset \Omega$ in the Hausdorff metric, where $P$ is a finite set of points;

(3) $u_{h} \rightarrow u$ in $L^{p}(\Omega)$ and $P_{\nabla u} \subseteq P$.

As in [9] we adopt the following ad hoc definition of $\Gamma$-convergence.

Definition 4.2. We say that $F_{\varepsilon} \Gamma$-converges to $\mathcal{F}$ if for every sequence of positive numbers $\left\{\varepsilon_{h}\right\}_{h} \rightarrow 0$ and for every $u \in \Delta \mathcal{A} \mathcal{M}^{p, 2}(\Omega)$ we have:

(1) for every sequence $\left\{\left(u_{h}, D_{h}\right)\right\}_{h} \subset Y(\Omega)$ H-converging to $u \in \Delta \mathcal{A} \mathcal{M}^{p, 2}(\Omega)$

$$
\liminf _{h \rightarrow+\infty} F_{\varepsilon_{h}}\left(u_{h}, D_{h}\right) \geq \mathcal{F}(u)
$$

(2) there exists a sequence $\left\{\left(u_{h}, D_{h}\right)\right\}_{h} \subset Y(\Omega)$ H-converging to $u$ such that

$$
\limsup _{h \rightarrow+\infty} F_{\varepsilon_{h}}\left(u_{h}, D_{h}\right) \leq \mathcal{F}(u) .
$$

We point out that with this approach, the Fundamental Theorem of the $\Gamma$-convergence cannot be applied directly, since we do not deal with a metric space (for a complete survey on $\Gamma$-convergence we refer to $[7,13]$ ). However it is still possible to prove that a sequence $\left\{\left(u_{h}, D_{h}\right)\right\}_{h}$ asymptotically minimizing $F_{\varepsilon}(u, D)$ admits a subsequence H-converging to a minimizer of $\mathcal{F}(u)$. Indeed we will show at the end of this section (see Thm. 4.4) that this property can be still obtained as a consequence of the compactness property of minimizing sequences of $F_{\varepsilon}, \Gamma$-liminf inequality (1) and $\Gamma$-limsup inequality (2).

\subsection{Compactness}

We state and prove the following compactness result.

Theorem 4.1. Let $\left\{\varepsilon_{h}\right\}_{h} \rightarrow 0^{+}$be such that

$$
F_{\varepsilon_{h}}\left(u_{h}, D_{h}\right) \leq M
$$

then there exist a subsequence $\left\{\left(u_{h_{k}}, D_{h_{k}}\right)\right\}_{k} \subset Y(\Omega)$, a function $u \in \Delta \mathcal{A M}^{p, 2}(\Omega)$ and a set $P \subset \bar{\Omega}$ of finite number of points, such that $\left\{\left(u_{h_{k}}, D_{h_{k}}\right)\right\}_{k} \mathrm{H}$-converges to $u$. 
Proof. We adapt an argument of [9]. From (4.5) we have immediately $\left\{D_{h}\right\} \subset R(\Omega)$ with $\mathcal{L}^{2}\left(D_{h}\right) \rightarrow 0$. Then we can parameterize every $C_{h}=\partial D_{h}$ by a finite and disjoint union of Jordan curves. Let us set for every $h$, $C_{h}=\bigcup_{i=1}^{m(h)} \gamma^{i}$. Then we have according to the 2-dimensional version of Gauss-Bonnet's Theorem and Young's inequality

$$
M \geq \frac{1}{4 \pi} \int_{\partial D_{h}}\left(\frac{1}{\varepsilon_{h}}+\varepsilon_{h} \kappa_{h}^{2}\right) \mathrm{d} \mathcal{H}^{1} \geq \frac{1}{4 \pi} \int_{\partial D_{h}} 2 \kappa_{h} \mathrm{~d} \mathcal{H}^{1}=\frac{1}{4 \pi} \int_{\bigcup_{i=1}^{m(h)} \gamma^{i}} 2 \kappa_{h} \mathrm{~d} \mathcal{H}^{1}=m(h) .
$$

Note that we have $m(h) \leq M$, with $M \geq 0$, independent of $h$. Then it is possible to extract a subsequence $C_{h_{k}}$ with the number of curves in $C_{h_{k}}$ equal to some $n$ for every $k$. Then we set $C_{h_{k}}=\left\{\gamma_{h_{k}}^{1}, \ldots, \gamma_{h_{k}}^{n}\right\}$ for any $k$. From (4.5) we also have for any $\gamma \in C_{h_{k}}$ that $\mathcal{H}^{1}(\gamma) \leq 4 \pi M \varepsilon_{h_{k}}$ and consequently $\max \left\{\mathcal{H}^{1}(\gamma): \gamma \in C_{h_{k}}\right\} \rightarrow 0$. Then there exists a finite set of point $P=\left\{x_{1}, \ldots, x_{n}\right\} \subset \bar{\Omega}$ such that for any radius $\rho$ there is an index $k_{\rho}$ with

$$
\gamma_{h_{k}}^{i} \subset B_{\rho}\left(x_{i}\right) \text { for all } k>k_{\rho} \text { and } i \in\{1, \ldots, n\},
$$

so that if we set $\partial D_{h_{k}}=\bigcup_{i=1}^{n} \gamma_{h_{k}}^{i} \subset \bigcup_{i=1}^{n} B_{\rho}\left(x_{i}\right)$, then the Hausdorff distance $d_{\mathrm{H}}\left(\partial D_{h_{k}}, P\right) \rightarrow 0$ since $\mathcal{L}^{2}\left(D_{h_{k}}\right) \rightarrow 0$, as $\rho \rightarrow 0$.

Now we prove the compactness property for $u_{h}$. First of all from the estimate

$$
\left\|\nabla u_{h}\right\|_{L^{p}(\Omega)}^{p} \leq 2^{p}\left(\left\|\nabla u_{h}-U_{0}\right\|_{L^{p}(\Omega)}^{p}+\left\|U_{0}\right\|_{L^{p}(\Omega)}^{p}\right)
$$

and (4.5), we may extract a subsequence $\left\{u_{h_{k}}\right\} \subset W_{0}^{1, p}(\Omega)$ weakly convergent to $u \in W_{0}^{1, p}(\Omega)$.

Let $\left\{\Omega_{j}\right\}_{j}$ be a sequence of open sets $\Omega_{j} \subset \subset \Omega \backslash P$ invading $\Omega \backslash P$. We claim that it is possible to extract a sequence of $D_{h_{k}}$ such that $\Omega_{j} \cap D_{h_{k}}=\emptyset$; for any $j$ and $k$ large enough. Indeed since the distance between $\Omega_{j}$ and $P$ is positive for any $j$ there exists $\eta_{j}$ such that $\Omega_{j} \cap\left(\bigcup_{i}^{n} B_{\eta_{j}}\left(x^{i}\right)\right)=\emptyset$. On the other hand we know that for every $\rho$ we can find $k_{\rho}$ such that $\partial D_{h_{k}}=\bigcup_{i=1}^{n} \gamma_{h_{k}}^{i} \subset \bigcup_{i=1}^{n} B_{\rho}\left(x_{i}\right)$, for every $k \geq k_{\rho}$. Then in particular if $\rho=\eta_{j}$ there exists $k_{j}$ such that for all $k \geq k_{j}$

$$
\Omega_{j} \cap \partial D_{h_{k}}=\emptyset,
$$

for all $j$. Therefore for any $x \in \Omega_{j}$ there exists $\delta>0$ such that either $B_{\delta}(x) \subset D_{h_{k}}$ or $B_{\delta}(x) \subset \Omega \backslash D_{h_{k}}$. Finally by taking into account that $\mathcal{L}^{2}\left(D_{h_{k}}\right) \rightarrow 0$ we conclude $\Omega_{j} \cap D_{h_{k}}=\emptyset$ for $k \geq k_{j}$.

Then for every $k \geq k_{j}$ we have that $u_{h_{k}} \in W^{1, p, 2}\left(\operatorname{div} ; \Omega_{j}\right)$ and by (4.5) we get

$$
\int_{\Omega_{j}}\left|\Delta u_{h_{k}}\right|^{2} \mathrm{~d} x \leq \int_{\Omega \backslash D_{h_{k}}}\left|\Delta u_{h_{k}}\right|^{2} \mathrm{~d} x \leq M
$$

Then we can extract a further subsequence still denoted by $\left\{u_{h_{k}}\right\} \subset W^{1, p, 2}\left(\operatorname{div} ; \Omega_{j}\right)$ such that

$$
\begin{cases}u_{h_{k}} \rightarrow u & \text { in } L^{p}\left(\Omega_{j} ; \mathbb{R}^{2}\right) \text { and a.e. } \\ \nabla u_{h_{k}} \rightarrow \nabla u & \text { in } L^{p}\left(\Omega_{j} ; \mathbb{R}^{2}\right) \\ \Delta u_{h_{k}} \rightarrow \Delta u & \text { in } L^{2}\left(\Omega_{j}\right)\end{cases}
$$

By standard diagonalization argument we may assume that the extracted subsequence is the same for every $j$.

Then by the semicontinuity of the $L^{2}$-norm we have

$$
\sup _{j} \int_{\Omega_{j}}|\Delta u|^{2} \mathrm{~d} x \leq \sup _{j} \liminf _{k+\infty} \int_{\Omega_{j}}\left|\Delta u_{k}\right|^{2} \mathrm{~d} x \leq M .
$$


If we set $\tilde{P}=P \backslash \partial \Omega$, then we deduce $u \in W_{0}^{1, p, 2}(\operatorname{div} ; \Omega \backslash \tilde{P})$ and therefore $\nabla u \in \operatorname{SDM}^{p}(\Omega)$ with $P_{\nabla u} \subseteq P$, by Proposition 3.1. So we conclude that $u \in \Delta \mathcal{A} \mathcal{M}^{p, 2}(\Omega)$.

\subsection{Lower bound}

We provide the lower bound (1) in Definition 4.2.

Theorem 4.2. Let $\left\{\varepsilon_{h}\right\}_{h}$ be a sequence of positive numbers converging to zero. For every sequence $\left\{\left(u_{h}, D_{h}\right)\right\}_{h} \subset$ $Y(\Omega)$, H-converging to $u \in \Delta \mathcal{A M}^{p, 2}(\Omega)$, we have

$$
\liminf _{h \rightarrow \infty} F_{\varepsilon_{h}}\left(u_{h}, D_{h}\right) \geq \mathcal{F}(u) .
$$

Proof. Up to a subsequence we may assume that the lim inf is actually a limit. As in the proof of Theorem 4.1, by setting for every $h, C_{h}=\bigcup_{i=1}^{m(h)} \gamma^{i}$, we get

$$
M \geq \frac{1}{4 \pi} \int_{\partial D_{h}}\left(\frac{1}{\varepsilon_{h}}+\varepsilon_{h} k^{2}\right) \mathrm{d} \mathcal{H}^{1}=m(h) .
$$

Up to subsequences we have $m(h)=n$ for some natural number $n$. Hence there exists a set $P$ of $n$ points such that $\partial D_{h}$ converges in the Hausdorff metric to $P$ with $P_{\nabla u} \subseteq P$.

Let now $\left\{\Omega_{j}\right\}_{j}$ be a sequence of open sets $\Omega_{j} \subset \subset \Omega \backslash P$ invading $\Omega \backslash P$. As in the proof of Theorem 4.1 we may assume, up to a subsequence, that $\Delta u_{h} \rightarrow \Delta u$ in $L^{2}\left(\Omega_{j}\right)$. Furthermore we have, by the same argument used in the proof of Theorem 4.1, $\Omega_{j} \subset \Omega \backslash D_{h}$ for h large and for any $j$. Consequently

$$
\liminf _{h \rightarrow+\infty} \int_{\Omega \backslash D_{h}}\left|\Delta u_{h}\right|^{2} \mathrm{~d} x \geq \liminf _{h \rightarrow+\infty} \int_{\Omega_{j}}\left|\Delta u_{h}\right|^{2} \mathrm{~d} x \geq \int_{\Omega_{j}}|\Delta u|^{2} \mathrm{~d} x .
$$

On the other hand, arguing as in Theorem 4.1, we infer that the limit $u$ of the subsequence $u_{h}$ belongs to $\Delta \mathcal{A} \mathcal{M}^{p, 2}(\Omega)$, with $\Delta u \in L^{2}(\Omega \backslash P)$ and $P_{\nabla u} \subseteq P$. So that by monotone convergence

$$
\liminf _{h \rightarrow+\infty} \int_{\Omega \backslash D_{h}}\left|\Delta u_{h}\right|^{2} \mathrm{~d} x \geq \int_{\Omega \backslash P}|\Delta u|^{2} \mathrm{~d} x=\int_{\Omega}|\Delta u|^{2} \mathrm{~d} x .
$$

As in the proof of Theorem 4.1, inequality (4.6) holds. Then we easily get

$$
\lim _{h \rightarrow \infty} \int_{\Omega}\left|\nabla u_{h}-U_{0}\right|^{p} \mathrm{~d} x \geq \int_{\Omega}\left|\nabla u-U_{0}\right|^{p} \mathrm{~d} x
$$

Finally we have

$$
\frac{1}{4 \pi} \int_{\partial D_{h}}\left(\frac{1}{\varepsilon_{h}}+\varepsilon_{h} k^{2}\right) \mathrm{d} \mathcal{H}^{1} \geq n=\mathcal{H}^{0}(P) \geq \mathcal{H}^{0}\left(P_{\nabla u}\right) .
$$

Eventually by (4.8), (4.9), (4.10) and by the superlinearity property of the lim inf operator we achieve the result.

\subsection{Upper bound}

In [9] for the construction of the optimal sequence it is crucial to use a result due to Chambolle and Doveri (see [10]). This result states that it is possible to approximate, in the $H^{1}$-norm, a function $u \in W^{1,2}(\Omega \backslash C)$ (where $C$ is a closed set), by means of a sequence of functions $u_{h} \in W^{1,2}\left(\Omega \backslash C_{h}\right)$ with $C_{h}$ convergent to $C$ in the Hausdorff metric. In our case this argument does not apply due to presence of a second order differential operator. Nevertheless since we work only with set of points it is possible to build an optimal sequence in a more direct way. 
Theorem 4.3. Let $\left\{\varepsilon_{h}\right\}_{h}$ be a sequence of positive numbers converging to 0 . For every $u \in \Delta \mathcal{A M} \mathcal{M}^{p, 2}(\Omega)$ there exists a sequence $\left\{\left(u_{h}, D_{h}\right)\right\}_{h} \subset Y(\Omega) \mathrm{H}$-converging to $u$ such that

$$
\limsup _{h \rightarrow+\infty} F_{\varepsilon_{h}}\left(u_{h}, D_{h}\right) \leq \mathcal{F}(u)
$$

Proof. We start by the construction of the sequence $D_{h}$. Let $n$ be the number of points $x_{i}$ in $P_{\nabla u}$. Then we take $D_{h}=\bigcup_{i=1}^{n} B_{\varepsilon_{h}}\left(x_{i}\right)$. So that $\mathcal{L}^{2}\left(D_{h}\right) \rightarrow 0, \frac{1}{\varepsilon_{h}} \mathcal{L}^{2}\left(D_{h}\right) \rightarrow 0$ and $\partial D_{h}$ converges with respect to the Hausdorff distance to $P_{\nabla u}$. Moreover for $h$ large enough we may assume $B_{\varepsilon_{h}}\left(x_{i}\right) \cap B_{\varepsilon_{h}}\left(x_{j}\right)=\emptyset$ for $i \neq j$. Now we build $u_{h}$. Let $\left\{\rho_{h}\right\} \subset \mathbb{R}$ be such that $\rho_{h} \geq 0$ and $\rho_{h} \rightarrow 0$ when $h \rightarrow \infty$. Let $\theta_{h} \in C^{\infty}(\Omega)$ with the following property:

$$
\begin{cases}\theta_{h}=1 & \text { on } B_{\frac{\rho_{h}}{2}}\left(x_{i}\right) \text { for any } i=1, \ldots, n \\ 0 \leq \theta_{h} \leq 1 & \text { on } B_{\rho_{h}}\left(x_{i}\right) \backslash B_{\frac{\rho_{h}}{2}}\left(x_{i}\right) \text { for any } i=1, \ldots, n \\ \theta=0 & \text { on } \Omega \backslash B_{\rho_{h}}\left(x_{i}\right) \text { for any } i=1, \ldots, n \\ \left\|\nabla \theta_{h}\right\|_{\infty} \leq \frac{1}{\rho_{h}} & \end{cases}
$$

We set $u_{h}=\left(1-\theta_{h}\right) u$. It is not difficult to check that $\left\{\left(u_{h}, D_{h}\right)\right\}_{h} \subset Y(\Omega)$ and H-converges to $u$. We claim that the pair $\left(u_{h}, D_{h}\right)$ realizes the inequality (4.11) for a suitable choice of the sequence $\rho_{h}$. By making the computation we have

Then

$$
\nabla u_{h}=\left(1-\theta_{h}\right) \nabla u-u \nabla \theta_{h} .
$$

so that

$$
\int_{\Omega}\left|\nabla u_{h}-U_{0}\right|^{p} \mathrm{~d} x=\int_{\Omega}\left|\nabla u-U_{0}-\theta_{h} \nabla u-u \nabla \theta_{h}\right|^{p} \mathrm{~d} x,
$$

$$
\limsup _{h \rightarrow+\infty} \int_{\Omega}\left|\nabla u_{h}-U_{0}\right|^{p} \mathrm{~d} x \leq \limsup _{h \rightarrow+\infty}\left(\left(\int_{\Omega}\left|\nabla u-U_{0}\right|^{p} \mathrm{~d} x\right)^{\frac{1}{p}}+\left(\int_{\Omega}\left|\theta_{h} \nabla u\right|^{p} \mathrm{~d} x\right)^{\frac{1}{p}}+\left(\int_{\Omega}\left|\nabla \theta_{h} u\right|^{p} \mathrm{~d} x\right)^{\frac{1}{p}}\right)^{p} .
$$

Since $|\nabla u|^{p} \in L^{1}(\Omega)$, we have by applying the dominated convergence theorem $\int_{\Omega}\left|\theta_{h} \nabla u\right|^{p} \mathrm{~d} x \rightarrow 0$. Let us focus on the term $\int_{\Omega}\left|\nabla \theta_{h} u\right|^{p}$. By the Sobolev embedding we have $u \in L^{p^{*}}(\Omega)$ with $p^{*}=\frac{2 p}{2-p}$ and hence $|u|^{p} \in L^{\frac{p^{*}}{p}}(\Omega)$, with $\frac{p^{*}}{p}=\frac{2}{2-p}$. have

By (4.12), using Holder's inequality with dual exponents $\frac{2}{2-p}$ and $\frac{2}{p}$, and taking into account that $p<2$, we

$$
\begin{aligned}
& \int_{\Omega}\left|\nabla \theta_{h} u\right|^{p} \mathrm{~d} x \leq \sum_{i=1}^{n} \int_{B_{\rho_{h}}\left(x_{i}\right) \backslash B_{\frac{\rho_{h}}{2}}\left(x_{i}\right)}\left|\nabla \theta_{h} u\right|^{p} \mathrm{~d} x=\sum_{i=1}^{n}\left(\int_{B_{\rho_{h}}\left(x_{i}\right)}\left|\nabla \theta_{h} u\right|^{p} \mathrm{~d} x-\int_{B_{\frac{\rho_{h}}{2}\left(x_{i}\right)}}\left|\nabla \theta_{h} u\right|^{p} \mathrm{~d} x\right) \\
& \leq \sum_{i=1}^{n}\left(\int_{B_{\rho_{h}}\left(x_{i}\right)}\left|\nabla \theta_{h}\right|^{2} \mathrm{~d} x\right)^{\frac{p}{2}}\|u\|_{L^{p^{*}\left(B_{\rho_{h}}\left(x_{i}\right)\right)}}^{p} \leq \sum_{i=1}^{n}\|u\|_{L^{p^{*}\left(B_{\rho_{h}}\left(x_{i}\right)\right)}}^{p} \pi \rightarrow 0,
\end{aligned}
$$

by Lebesgue's Theorem, since the sequence $\chi_{B_{\rho_{h}}\left(x_{i}\right)}$ converges to 0 almost everywhere. From (4.13) it follows that

$$
\begin{aligned}
\limsup _{h \rightarrow+\infty} \int_{\Omega}\left|\nabla u_{h}-U_{0}\right|^{p} \mathrm{~d} x & \leq \lim _{h \rightarrow+\infty}\left(\left(\int_{\Omega}\left|\nabla u-U_{0}\right|^{p} \mathrm{~d} x\right)^{\frac{1}{p}} \mid+\left(\int_{\Omega}\left|\theta_{h} \nabla u\right|^{p} \mathrm{~d} x\right)^{\frac{1}{p}}+\left(\int_{\Omega}\left|\nabla \theta_{h} u\right|^{p} \mathrm{~d} x\right)^{\frac{1}{p}}\right)^{p} \\
& =\left(\left(\int_{\Omega}\left|\nabla u-U_{0}\right|^{p} \mathrm{~d} x\right)^{\frac{1}{p}}\right)^{p}=\int_{\Omega}\left|\nabla u-U_{0}\right|^{p} \mathrm{~d} x
\end{aligned}
$$


Now we compute $\Delta u_{h}$. The identity $\operatorname{div}(f A)=f \operatorname{div} A+\nabla f \cdot A$ yields

$$
\Delta u_{h}=\left(1-\theta_{h}\right) \Delta u-2 \nabla \theta_{h} \nabla u-\Delta \theta_{h} u .
$$

Then by choosing $\rho_{h}$ small enough we have from (4.12)

$$
\limsup _{h \rightarrow+\infty} \int_{\Omega \backslash D_{h}}\left|\Delta u_{h}\right|^{2} \mathrm{~d} x \leq \lim _{h \rightarrow+\infty} \int_{\Omega \backslash D_{h}}|\Delta u|^{2} \mathrm{~d} x=\int_{\Omega}|\Delta u|^{2} \mathrm{~d} x .
$$

Finally since for $h$ large we have $B_{\varepsilon_{h}}\left(x_{i}\right) \cap B_{\varepsilon_{h}}\left(x_{j}\right)=\emptyset$ for $i \neq j$ we get

$$
\lim _{h} \frac{1}{4 \pi} \int_{\partial D_{h}}\left(\frac{1}{\varepsilon_{h}}+\varepsilon_{h} k^{2}\right) \mathrm{d} \mathcal{H}^{1}=\lim _{h} \sum_{i=1}^{n} \frac{1}{4 \pi} \int_{\partial B_{\varepsilon_{h}}\left(x_{i}\right)} \frac{2}{\varepsilon_{h}} \mathrm{~d} \mathcal{H}^{1}=n=\mathcal{H}^{0}\left(P_{\nabla u}\right) .
$$

By recalling that the limsup is a sublinear operation, by (4.15)-(4.17), we achieve the result.

\subsection{Variational property}

We conclude this section by properly stating and proving the particular version of the Fundamental Theorem of $\Gamma$-convergence which is, in this case, a direct consequence of Theorems 4.1, 4.2 and 4.3. The proof can be achieved by a classical argument (see [7], Sect. 1.5). However we prefer to give the proof in order to make clear that the classical variational setting is not directly available, and therefore the variational property has to be proven.

Theorem 4.4. Let $F_{\varepsilon}$ and $\mathcal{F}$ be given respectively by (4.4) and (4.3). If $\left\{\varepsilon_{h}\right\}_{h}$ is a sequence of positive numbers converging to zero and $\left\{\left(u_{h}, D_{h}\right)\right\} \subset Y(\Omega)$ such that

$$
\lim _{h \rightarrow+\infty}\left(F_{\varepsilon_{h}}\left(u_{h}, D_{h}\right)-\inf _{Y(\Omega)} F_{\varepsilon_{h}}(u, D)\right)=0,
$$

then there exist a subsequence $\left\{\left(u_{h_{k}}, D_{h_{k}}\right)\right\} \subset Y(\Omega)$ and a minimizer $\bar{u}$ of $\mathcal{F}(u)$ with $\bar{u} \in \Delta \mathcal{A} \mathcal{M}^{p, 2}(\Omega)$, such that $\left\{\left(u_{h_{k}}, D_{h_{k}}\right)\right\} \mathrm{H}$-converges to $\bar{u}$.

Proof. We know from Theorems 4.2 and 4.3 that $F_{\varepsilon} \Gamma$-converges to $\mathcal{F}$. Let $\delta>0$ and $u \in \Delta \mathcal{A M}^{p, 2}(\Omega)$ be such that

$$
\mathcal{F}(u) \leq \inf _{\Delta \mathcal{A} \mathcal{M}^{p, 2}(\Omega)} \mathcal{F}(u)+\delta .
$$

From Theorem 4.3 there exists a sequence $\left\{\left(\tilde{u_{h}}, \tilde{D_{h}}\right)\right\} \subset Y(\Omega)$ such that

$$
\inf _{\Delta \mathcal{A} \mathcal{M}^{p, 2}(\Omega)} \mathcal{F}+\delta \geq \mathcal{F}(u) \geq \limsup _{h \rightarrow+\infty} F_{\varepsilon_{h}}\left(\tilde{u_{h}}, \tilde{D_{h}}\right) .
$$

Then since $\delta$ is arbitrary it follows that

$$
\limsup _{h \rightarrow+\infty} \inf _{Y(\Omega)} F_{\varepsilon_{h}} \leq \limsup _{h \rightarrow+\infty} F_{\varepsilon_{h}}\left(\tilde{u_{h}}, \tilde{D_{h}}\right) \leq \inf _{\Delta \mathcal{A M}^{p, 2}(\Omega)} \mathcal{F} .
$$

Let now $\left\{\left(u_{h}, D_{h}\right)\right\} \subset Y(\Omega)$ be such that $\lim _{h \rightarrow+\infty}\left(F_{\varepsilon_{h}}\left(u_{h}, D_{h}\right)-\inf _{Y(\Omega)} F_{\varepsilon_{h}}(u, D)\right)=0$. Then from Theorem 4.1, up to subsequences, the sequence $\left\{\left(u_{h}, D_{h}\right)\right\}_{h}$ H-converges to some $\bar{u} \in \Delta \mathcal{A M}^{p, 2}(\Omega)$. Then by Theorem 4.2 and taking into account (4.18) we deduce

$$
\inf _{\Delta \mathcal{A} \mathcal{M}^{p, 2}(\Omega)} \mathcal{F} \leq \mathcal{F}(\bar{u}) \leq \liminf _{h \rightarrow+\infty} \inf _{Y(\Omega)} F_{\varepsilon_{h}} \leq \limsup _{h \rightarrow+\infty} \inf _{Y(\Omega)} F_{\varepsilon_{h}} \leq \inf _{\Delta \mathcal{A} \mathcal{M}^{p, 2}(\Omega)} \mathcal{F}
$$

Then we easily get the thesis. 


\section{Approximation by Smooth FunCtions}

By following the Braides-March's approach in [9] we approximate the measure $\mathcal{H}^{1}\lfloor\partial D$ by the ModicaMortola's energy density given by $\left(\varepsilon|\nabla w|^{2}+\frac{1}{\varepsilon} W(w)\right) \mathrm{d} x$ where $W(w)=w^{2}(1-w)^{2}$ and $w \in C^{\infty}(\Omega)$. The next step is to replace the regular set $D$ with the level set of $w$. Let us set $Z=\{x, \nabla w(x)=0\}$. By Sard's Lemma we have that $\mathcal{L}^{1}(w(Z))=0$. In particular, if $w$ takes values into the interval $[0,1]$, we infer that for almost every $t \in(0,1)$ the set $Z \cap w^{-1}(t)$ is empty. Consequently for almost every $t \in(0,1)$ the $t$-level set $\{w<t\}$ is a regular set with boundary $\{w=t\}$. Now, since we want to replace the set $D$, we need that $\{w<t\} \subset \subset \Omega$. Then we require $1-w \in C_{0}^{\infty}(\Omega ;[0,1])$. Furthermore for almost every $t$, we have $k(\{w=t\})=\operatorname{div}\left(\frac{\nabla w}{|\nabla w|}\right)$, where $k$ is the curvature of the set $\{w=t\}$. From all of this we are led to define the following space:

$$
S(\Omega)=\left\{(u, w) ; u \in W_{0}^{1, p, 2}(\operatorname{div} ; \Omega) ; 1-w \in C_{0}^{\infty}(\Omega ;[0,1])\right\}
$$

and having in mind the coarea formula, the following sequence of functionals defined on $S(\Omega)$

$$
\begin{aligned}
\mathcal{G}_{\varepsilon}(u, w)= & \int_{\Omega} w^{2}|\Delta u|^{2} \mathrm{~d} x+\frac{1}{8 \pi C} \int_{\Omega \backslash\{|\nabla w|=0\}}\left(\frac{1}{\beta_{\varepsilon}}+\beta_{\varepsilon}\left(\operatorname{div}\left(\frac{\nabla w}{|\nabla w|}\right)\right)^{2}\right)\left(\varepsilon|\nabla w|^{2}+\frac{1}{\varepsilon} W(w)\right) \mathrm{d} x \\
& +\int_{\Omega}\left|\nabla u-U_{0}\right|^{p} \mathrm{~d} x+\frac{1}{\gamma_{\varepsilon}} \int_{\Omega}(1-w)^{2} \mathrm{~d} x
\end{aligned}
$$

with $C=\int_{0}^{1} \sqrt{W(t)} \mathrm{d} t$. The last term forces $w_{\varepsilon}$ to be equal to one almost everywhere in the limit. From now on, the parameters $\varepsilon, \beta_{\varepsilon}, \gamma_{\varepsilon}$ will be related as follows:

$$
\begin{gathered}
\lim _{\varepsilon \rightarrow 0^{+}} \frac{\beta_{\varepsilon}}{\gamma_{\varepsilon}}=0, \\
\lim _{\varepsilon \rightarrow 0^{+}} \frac{\varepsilon|\log (\varepsilon)|}{\beta_{\varepsilon}}=0 .
\end{gathered}
$$

The convergence that plays the role of the $\mathrm{H}$-convergence is the following. With a slight abuse of notation this convergence will be still denoted by $\mathrm{H}$.

Definition 5.1. Let $\left\{\left(u_{h}, w_{h}\right)\right\}_{h}$ be a sequence $S(\Omega)$. Set $D_{h}^{t}=\left\{w_{h}<t\right\}$. We say that $\left\{\left(u_{h}, w_{h}\right)\right\}_{h}$ H-converges to $u \in \Delta \mathcal{A M}^{p, 2}(\Omega)$, as $h \rightarrow+\infty$, if for every $t \in(0,1)$ the sequence $\left\{\left(u_{h}, D_{h}^{t}\right)\right\}_{h}$ in $Y(\Omega)$ H-converges to $u$.

As in the previous section, we adopt the $a d$ hoc definition of $\Gamma$-convergence with respect to the convergence above.

Definition 5.2. We say that $\mathcal{G}_{\varepsilon} \Gamma$-converges to $\mathcal{F}$ if, for every sequence of positive numbers $\varepsilon_{h} \rightarrow 0$ and for every $u \in \Delta \mathcal{A M}^{p, 2}(\Omega)$, we have:

(1) for every sequence $\left\{\left(u_{h}, w_{h}\right)\right\}_{h} \subset S(\Omega)$ H-converging to $u$

$$
\liminf _{h \rightarrow+\infty} \mathcal{G}_{\varepsilon_{h}}\left(u_{h}, w_{h}\right) \geq \mathcal{F}(u)
$$

(2) there exists a sequence $\left\{\left(u_{h}, w_{h}\right)\right\}_{h} \subset S(\Omega)$ H-converging to $u$ such that

$$
\limsup _{h \rightarrow+\infty} \mathcal{G}_{\varepsilon_{h}}\left(u_{h}, w_{h}\right) \leq F(u) .
$$

As in the previous section, we remark that the property which guarantees the convergence of the sequences asymptotically minimizing $\mathcal{G}_{\varepsilon}$ to a minimum of $\mathcal{F}$ must be proved, since we cannot apply the Fundamental Theorem of $\Gamma$-convergence. We will state the analogous of Theorem 4.4 at the end of the section. 


\subsection{Compactness}

The compactness result goes as follows.

Theorem 5.1. Let $\left\{\varepsilon_{h}\right\}_{h} \rightarrow 0^{+}$such that

$$
F_{\varepsilon_{h}}\left(u_{h}, w_{h}\right) \leq M
$$

Then there exist a subsequence $\left\{\left(u_{h_{k}}, w_{h_{k}}\right)\right\}_{k} \subset S(\Omega), u \in \Delta \mathcal{A M}^{p, 2}(\Omega)$ such that $\left\{\left(u_{h_{k}}, w_{h_{k}}\right)\right\}_{k} \mathrm{H}$-converges to $u$.

Proof. The first part of proof is as in [9]. For the convenience of the reader we give the complete proof.

By Young's inequality and by (5.5) we get

$$
M \geq 2 \int_{\Omega \backslash\left\{\left|\nabla w_{h}\right|=0\right\}}\left|\nabla w_{h}\right| \sqrt{W\left(w_{h}\right)}\left(\frac{1}{\beta_{\varepsilon_{h}}}+\beta_{\varepsilon_{h}}\left(\operatorname{div}\left(\frac{\nabla w_{h}}{\left|\nabla w_{h}\right|}\right)\right)^{2}\right) \mathrm{d} x .
$$

Now by coarea formula, we obtain

$$
M \geq 2 \int_{0}^{1} \sqrt{W(t)} \int_{\left\{w_{h}=t\right\} \cap\left\{\left|\nabla w_{h}\right| \neq 0\right\}}\left(\frac{1}{\beta_{\varepsilon_{h}}}+\beta_{\varepsilon_{h}}\left(\operatorname{div}\left(\frac{\nabla w_{h}}{\left|\nabla w_{h}\right|}\right)\right)^{2}\right) \mathrm{d} \mathcal{H}^{1} \mathrm{~d} t .
$$

Thanks to Sard's Lemma, for any $h$ there exists a $\mathcal{L}^{1}$-negligible set $\mathcal{N}_{w_{h}} \subseteq(0,1)$ such that

$$
\left\{w_{h}=t\right\}=\partial\left\{w_{h}<t\right\},\left\{w_{h}<t\right\} \in R(\Omega), \text { for } t \in(0,1) \backslash \mathcal{N}_{w_{h}} .
$$

On $\left\{w_{h}=t\right\}$ for $t \in(0,1) \backslash \mathcal{N}_{w_{h}}$ we have

$$
\left|\nabla w_{h}\right| \neq 0 \text { and } \kappa\left(\left\{w_{h}=t\right\}\right)=\operatorname{div}\left(\frac{\nabla w_{h}}{\left|\nabla w_{h}\right|}\right) .
$$

Now since the union $\bigcup_{h} \mathcal{N}_{w_{h}}$ is $\mathcal{L}^{1}$-negligible, from (5.6) we have

$$
M \geq 2 \int_{(0,1) \backslash \cup_{h} \mathcal{N}_{w_{h}}} \sqrt{W(t)} \int_{\partial\left\{w_{h}<t\right\}}\left(\frac{1}{\beta_{\varepsilon_{h}}}+\beta_{\varepsilon_{h}} \kappa^{2}\right) \mathrm{d} \mathcal{H}^{1} \mathrm{~d} t .
$$

By applying Fatou's Lemma and taking into account that the set $\bigcup_{h} \mathcal{N}_{w_{h}}$ does not depend on $h$ we get

$$
M \geq 2 \int_{(0,1) \backslash \bigcup_{h} \mathcal{N}_{w_{h}}} \sqrt{W(t)} \liminf _{h \rightarrow+\infty} \int_{\partial\left\{w_{h}<t\right\}}\left(\frac{1}{\beta_{\varepsilon_{h}}}+\beta_{\varepsilon_{h}} \kappa^{2}\right) \mathrm{d} \mathcal{H}^{1} \mathrm{~d} t
$$

Hence we deduce the existence of a $\mathcal{L}^{1}$-negligible set $Q$, with $\bigcup_{h} \mathcal{N}_{w_{h}} \subseteq Q$, such that for every $t \in(0,1) \backslash Q$

$$
\liminf _{h \rightarrow+\infty} \int_{\partial\left\{w_{h}<t\right\}}\left(\frac{1}{\beta_{\varepsilon_{h}}}+\beta_{\varepsilon_{h}} \kappa^{2}\right) \mathrm{d} \mathcal{H}^{1} \leq M_{t}
$$

where the constant $M_{t}$ does not depend on $h$.

Then, by the same density argument used in the proof of Theorem 5.1 of [9], by taking the limit $\rho \rightarrow 0^{+}$we infer $\partial\left\{w_{h_{k}}<t\right\}$ converges with respect to the Hausdorff metric to a set $P^{t} \subset \bar{\Omega}$ for every $t \in(0,1)$. Therefore, as in the proof of Theorem 4.1, we can extract a subsequence $\left\{u_{h_{k}}\right\}_{k}$ which converges strongly in $L^{p}(\Omega)$ to a function $u \in \Delta \mathcal{A M}^{p, 2}(\Omega)$ with $P_{\nabla u} \subseteq P^{t}$ for every $t \in(0,1)$. Hence we have that for every $t \in(0,1)$ the sequence $\left\{\left(u_{h_{k}}, D_{h_{k}}^{t}\right)\right\}_{k} \mathrm{H}$-converges to $u$ and the proof is achieved. 


\subsection{Lower bound}

We give the proof of the lower bound (1) in Definition 5.2. In the proof it will be crucial having the convergence of the $t$-level set for every $t \in(0,1)$.

Theorem 5.2. Let $\left\{\varepsilon_{h}\right\}_{h}$ be a sequence of positive numbers converging to zero. For every sequence $\left\{\left(u_{h}, w_{h}\right)\right\}_{h} \subset$ $S(\Omega) \mathrm{H}$-converging to $u \in \Delta \mathcal{A M}^{p, 2}(\Omega)$, we have

$$
\liminf _{h \rightarrow+\infty} F_{\varepsilon_{h}}\left(u_{h}, w_{h}\right) \geq \mathcal{F}(u) .
$$

Proof. Without loss of generality we assume, up to subsequences,

$$
+\infty>\liminf _{h \rightarrow+\infty} \mathcal{F}_{\varepsilon_{h}}=\lim _{h \rightarrow+\infty} \mathcal{F}_{\varepsilon_{h}}
$$

As in the proof of Theorem 5.1 we get that, for every $t \in(0,1), \mathcal{L}^{2}\left(\left\{w_{h}<t\right\}\right) \rightarrow 0$ and $\partial\left\{w_{h}<t\right\} \rightarrow P^{t}$ in the Hausdorff distance. For any $t \in(0,1)$ we have (see also [9] for a similar argument)

$$
\int_{\Omega} w_{h}{ }^{2}\left|\Delta u_{h}\right|^{2} \mathrm{~d} x=\int_{\left\{w_{h}<t\right\}} w_{h}{ }^{2}\left|\Delta u_{h}\right|^{2} \mathrm{~d} x+\int_{\left\{w_{h} \geq t\right\}} w_{h}{ }^{2}\left|\Delta u_{h}\right|^{2} \mathrm{~d} x \geq t^{2} \int_{\Omega}\left(1-\chi_{\left\{w_{h}<t\right\}}\right)\left|\Delta u_{h}\right|^{2} \mathrm{~d} x
$$

Let $\left\{\Omega_{j}\right\}_{j}$ be a sequence of open sets $\Omega_{j} \subset \subset \Omega \backslash P^{t}$ invading $\Omega \backslash P^{t}$. Then we may assume that $u_{h} \rightarrow$ weakly in $W_{0}^{1, p}(\Omega)$ and $\Delta u_{h}$ converges weakly in $L^{2}\left(\Omega_{j}\right)$ to $\Delta u$. Therefore as in the proof of Theorem 4.2 we get

$$
\lim _{h \rightarrow+\infty} \int_{\Omega}\left|\nabla u_{h}-U_{0}\right|^{p} \mathrm{~d} x \geq \int_{\Omega}\left|\nabla u-U_{0}\right|^{p} \mathrm{~d} x
$$

and

$$
\liminf _{h \rightarrow+\infty} t^{2} \int_{\Omega}\left(1-\chi_{\left\{w_{h}<t\right\}}\right)\left|\Delta u_{h}\right|^{2} \mathrm{~d} x \geq t^{2} \int_{\Omega_{j}}|\Delta u|^{2} \mathrm{~d} x
$$

for any $j$.

Then by (5.9) and, by taking into account that $|\Delta u|$ is in $L^{2}\left(\Omega \backslash P^{t}\right)$ with $P_{\nabla u} \subseteq P^{t}$, it follows that

$$
\liminf _{h \rightarrow+\infty} \int_{\Omega} w_{h}^{2}\left|\Delta u_{h}\right|^{2} \mathrm{~d} x \geq t^{2} \int_{\Omega}|\Delta u|^{2} \mathrm{~d} x
$$

and eventually by taking the limit $t \rightarrow 1$

$$
\liminf _{h \rightarrow+\infty} \int_{\Omega} w_{h}{ }^{2}\left|\Delta u_{h}\right|^{2} \mathrm{~d} x \geq \int_{\Omega}|\Delta u|^{2} \mathrm{~d} x .
$$

Finally, as in the proof of Theorem 4.2 (inequality 4.10) we have

$$
\liminf _{h \rightarrow+\infty} \frac{1}{4 \pi} \int_{\partial\left\{w_{h}<t\right\}}\left(\frac{1}{\beta_{\varepsilon_{h}}}+\beta_{\varepsilon_{h}} k^{2}\right) \mathrm{d} \mathcal{H}^{1} \geq \mathcal{H}^{0}\left(P^{t}\right) \geq \mathcal{H}^{0}\left(P_{\nabla u}\right) .
$$


Now arguing as in the proof of Theorem 5.1 and by taking into account (5.12), we get

$$
\begin{aligned}
& \liminf _{h \rightarrow+\infty} \int_{\Omega \backslash\left\{\left|\nabla w_{h}\right|=0\right\}}\left(\frac{1}{\beta_{\varepsilon_{h}}}+\beta_{\varepsilon_{h}}\left(\operatorname{div}\left(\frac{\nabla w_{h}}{\left|\nabla w_{h}\right|}\right)\right)^{2}\right)\left(\varepsilon_{h}\left|\nabla w_{h}\right|^{2}+\frac{1}{\varepsilon_{h}} W\left(w_{h}\right)\right) \mathrm{d} x \\
\geq & 2 \liminf _{h \rightarrow+\infty} \int_{(0,1) \backslash \cup_{h} \mathcal{N}_{w_{h}}} \sqrt{W(t)} \liminf _{h \rightarrow+\infty} \int_{\partial\left\{w_{h}<t\right\}}\left(\frac{1}{\beta_{\varepsilon_{h}}}+\beta_{\varepsilon_{h}} k^{2}\right) \mathrm{d} \mathcal{H}^{1} \mathrm{~d} t \\
\geq & 8 \pi \int_{(0,1)} \mathcal{H}^{0}\left(P^{t}\right) \sqrt{W(t)} \mathrm{d} t \geq 8 \pi C \mathcal{H}^{0}\left(P_{\nabla u}\right) .
\end{aligned}
$$

By collecting (5.10), (5.11) and (5.13) we achieve the thesis.

\subsection{Upper bound}

As in [9] to build $w_{h}$ we use the construction given in [4], while the optimal sequence $u_{k}$ is chosen as in Theorem 4.3.

Theorem 5.3. Let $\left\{\varepsilon_{h}\right\}$ be a sequence of positive numbers converging to 0 . For every $u \in \Delta \mathcal{A M}^{p, 2}$ there exists a sequence $\left\{\left(u_{h}, w_{h}\right)\right\}_{h} \subset S(\Omega)$, H-converging to $u$, such that

$$
\limsup _{h \rightarrow \infty} \mathcal{G}_{\varepsilon_{h}}\left(u_{h}, w_{h}\right) \leq \mathcal{F}(u) .
$$

Proof. If $A \subset \mathbb{R}^{2}$ we set

$$
\delta_{A}(x)=d(x, A)-d\left(x, \mathbb{R}^{2} \backslash A\right) .
$$

We start with the construction of $w_{h}$.

As in the proof of Theorem 4.3 we set $P_{\nabla u}=\left\{x_{1}, \ldots, x_{n}\right\}$ and we define

$$
D_{h}=\bigcup_{i=1}^{n} B_{\beta_{\varepsilon_{h}}}\left(x_{i}\right)
$$

Since $D_{h}$ is a regular set by taking into account condition (5.4) for $h$ large enough we have

$$
\left\{x \in \Omega: d\left(x, D_{h}\right)<2 \varepsilon_{h}\left|\log \varepsilon_{h}\right|\right\} \subset \subset \Omega .
$$

Let $\eta$ be the optimal profile for Modica-Mortola's energy, that is the solution of the ODE

$$
\left\{\begin{array}{l}
\eta^{\prime}(t)=\sqrt{W(\eta(t))} \quad \text { on } \mathbb{R} \\
\eta(-\infty)=0 \\
\eta(+\infty)=1
\end{array}\right.
$$

given by $\eta(t)=\frac{1}{2}\left(1+\tanh \frac{t}{2}\right)$.

For every $h$ let $\psi_{h}:[0,+\infty) \rightarrow[0,1]$ be a $C^{\infty}$-function such that

$$
\begin{cases}\psi_{h}=1 & \text { on }\left[0,\left|\log \varepsilon_{h}\right|\right] \\ \psi_{h}=0 & \text { on }\left[2\left|\log \varepsilon_{h}\right|,+\infty\right] \\ \psi_{h}^{\prime}<0 & \text { on }\left[\left|\log \varepsilon_{h}\right|, 2\left|\log \varepsilon_{h}\right|\right] \\ \left\|\psi_{h}^{\prime}\right\|_{L^{\infty}\left(\left|\log \varepsilon_{h}\right|, 2\left|\log \varepsilon_{h}\right|\right)}=O\left(\frac{1}{\left|\log \varepsilon_{h}\right|}\right) & \end{cases}
$$


As in [4] and in [9] we define

$$
\eta_{h}(t)= \begin{cases}\eta\left(\frac{t}{\varepsilon_{h}}\right) \psi_{h}\left(\frac{t}{\varepsilon_{h}}\right)+1-\psi_{h}\left(\frac{t}{\varepsilon_{h}}\right) & \text { if } t \geq 0 \\ 1-\eta\left(\frac{-t}{\varepsilon_{h}}\right) & \text { if } t<0 .\end{cases}
$$

Then we set $w_{h}(x)=\eta_{h}\left(\delta_{D_{h}}(x)\right)$. We claim that $1-w_{h}(x) \in C_{0}^{\infty}(\Omega ;[0,1])$ for $h$ large enough. It is not difficult to check that $w_{h} \in C_{0}^{\infty}(\Omega)$. Let now $x \in \partial \Omega$ then $\delta_{D_{h}}(x) \geq 0$ and $1-w_{h}(x)=\psi_{h}\left(\frac{\delta_{D_{h}}(x)}{\varepsilon_{h}}\right)\left(1-\eta\left(\frac{\delta_{D_{h}}(x)}{\varepsilon_{h}}\right)\right)$. From (5.15), it follows $\delta_{D_{h}}(x) \geq 2 \varepsilon_{h}\left|\log \varepsilon_{h}\right|$ for $h$ large enough, hence the claim follows. Then we take $\left\{\left(u_{h}, w_{h}\right)\right\}_{h}$ as optimal sequence, where $u_{h}$ is given as in Theorem 4.3.

First of all we have to check that $\left\{\left(u_{h}, w_{h}\right)\right\}_{h}$ H-converges to $u$. For any $x \in \Omega \backslash P_{\nabla u}$ we have that for $h$ large enough $\delta_{D_{h}}(x) \geq 0$ and one can check that $w_{h}(x) \rightarrow 1$ for every $x \in \Omega \backslash P_{\nabla u}$. This implies that $\mathcal{L}^{2}\left(\left\{w_{h}<t\right\}\right) \rightarrow 0$ for every $t \in(0,1)$. Now for every $t \in(0,1)$ we write

$$
\left\{w_{h}=t\right\}=\left(\left\{w_{h}=t\right\} \cap D_{h}\right) \cup\left(\left\{w_{h}=t\right\} \cap \Omega \backslash D_{h}\right) .
$$

Hence, since $w_{h}(x) \rightarrow 1$ for $x \in \Omega \backslash P_{\nabla u}$, for any $t \in(0,1)$ there exists $h(t)$ such that $\left\{w_{h}=t\right\} \cap \Omega \backslash D_{h}=\emptyset$ for every $h \geq h(t)$. So that from (5.16) it follows that for every $t \in(0,1),\left\{w_{h}=t\right\} \rightarrow P_{\nabla u}$ when $h \rightarrow+\infty$. So we can conclude that $\left(u_{h}, w_{h}\right) \mathrm{H}$-converges to $u$.

As in [4] we set

$$
D_{h}^{1}=\left\{x \in \Omega:\left|\delta_{D_{h}}(x)\right|<\varepsilon_{h}\left|\log \varepsilon_{h}\right|\right\}, D_{h}^{2}=\left\{x \in \Omega: \varepsilon_{h}\left|\log \varepsilon_{h}\right|<\left|\delta_{D_{h}}(x)\right|<2 \varepsilon_{h}\left|\log \varepsilon_{h}\right|\right\} .
$$

Therefore we can write

$$
\begin{aligned}
\int_{\Omega \backslash\left\{\left|\nabla w_{h}\right|=0\right\}} & \left(\frac{1}{\beta_{\varepsilon_{h}}}+\beta_{\varepsilon_{h}}\left(\operatorname{div}\left(\frac{\nabla w_{h}}{\left|\nabla w_{h}\right|}\right)\right)^{2}\right)\left(\varepsilon_{h}\left|\nabla w_{h}\right|^{2}+\frac{1}{\varepsilon_{h}} W\left(w_{h}\right)\right) \mathrm{d} x \\
= & \int_{D_{h}^{1} \backslash\left\{\left|\nabla w_{h}\right|=0\right\}}\left(\frac{1}{\beta_{\varepsilon_{h}}}+\beta_{\varepsilon_{h}}\left(\operatorname{div}\left(\frac{\nabla w_{h}}{\left|\nabla w_{h}\right|}\right)\right)^{2}\right)\left(\varepsilon_{h}\left|\nabla w_{h}\right|^{2}+\frac{1}{\varepsilon_{h}} W\left(w_{h}\right)\right) \mathrm{d} x \\
& +\int_{D_{h}^{2} \backslash\left\{\left|\nabla w_{h}\right|=0\right\}}\left(\frac{1}{\beta_{\varepsilon_{h}}}+\beta_{\varepsilon_{h}}\left(\operatorname{div}\left(\frac{\nabla w_{h}}{\left|\nabla w_{h}\right|}\right)\right)^{2}\right)\left(\varepsilon_{h}\left|\nabla w_{h}\right|^{2}+\frac{1}{\varepsilon_{h}} W\left(w_{h}\right)\right) \mathrm{d} x \\
= & I_{h}+I I_{h} .
\end{aligned}
$$

For $x \in D_{h}^{1}$, we have $\frac{\left|\delta_{D_{h}}(x)\right|}{\varepsilon_{h}}<\left|\log \varepsilon_{h}\right|$ therefore $w_{h}(x)=\eta\left(\frac{\left|\delta_{D_{h}(x)}\right|}{\varepsilon_{h}}\right)$. By taking into account the definition of $\eta$ we have $\frac{\varepsilon_{h}}{1+\varepsilon_{h}} \leq w_{h}(x) \leq \frac{1}{1+\varepsilon_{h}}$. Moreover it easy to check that

$$
\eta_{h}^{\prime}(t)=\frac{1}{\varepsilon_{h}} \eta^{\prime}\left(\frac{t}{\varepsilon_{h}}\right)=\frac{1}{\varepsilon_{h}} \sqrt{W \eta\left(\left(\frac{t}{\varepsilon_{h}}\right)\right)} ; \quad\left|\nabla w_{h}(x)\right|=\left|\eta_{h}^{\prime}\left(\delta_{D_{h}(x)}\right)\right| .
$$

This, together with the coarea formula yields

$$
I_{h}=2 \int_{\frac{\varepsilon_{h}}{1+\varepsilon_{h}}}^{\frac{1}{1+\varepsilon_{h}}} \sqrt{W(t)} \int_{\left\{w_{h}=t\right\}}\left(\frac{1}{\beta_{h}}+\beta_{h} k^{2}\right) \mathrm{d} \mathcal{H}^{1} \mathrm{~d} t .
$$


Now by change of variables we get

$$
I_{h}=2 \int_{-\varepsilon_{h}\left|\log \varepsilon_{h}\right|}^{\varepsilon_{h}\left|\log \varepsilon_{h}\right|} \eta_{h}^{\prime} \sqrt{W\left(\eta_{h}(s)\right)} \int_{\left\{\delta_{D_{h}}=s\right\}}\left(\frac{1}{\beta_{h}}+\beta_{h} k^{2}\right) \mathrm{d} \mathcal{H}^{1} \mathrm{~d} s
$$

Since for $h$ large enough we have (see the proof of [4], Thm. 4.3)

$$
\int_{\left\{\delta_{D_{h}}=s\right\}}\left(\frac{1}{\beta_{h}}+\beta_{h} k^{2}\right) \mathrm{d} \mathcal{H}^{1}=\int_{\partial D_{h}}\left(\frac{1}{\beta_{h}}+\beta_{h} k^{2}\right) \mathrm{d} t+O\left(\varepsilon_{h}\left|\log \left(\varepsilon_{h}\right)\right|\right),
$$

we get

$$
I_{h}=2 \int_{\frac{\varepsilon_{h}}{1+\varepsilon_{h}}}^{\frac{1}{1+\varepsilon_{h}}} \sqrt{W(t)} \mathrm{d} t \int_{\partial D_{h}}\left(\frac{1}{\beta_{h}}+\beta_{h} k^{2}\right) \mathrm{d} \mathcal{H}^{1}+O\left(\varepsilon_{h}\left|\log \left(\varepsilon_{h}\right)\right|\right) \int_{\frac{\varepsilon_{h}}{1+\varepsilon_{h}}}^{\frac{1}{1+\varepsilon_{h}}} \sqrt{W(t)} \mathrm{d} t .
$$

Then by using (4.17)

$$
\lim _{h \rightarrow+\infty} I_{h}=8 \pi \mathcal{H}^{0}\left(P_{\nabla u}\right) \int_{0}^{1} \sqrt{W(t)} \mathrm{d} t
$$

Moreover with the same argument and by using the definition of $w_{h}$ one can check that

$$
\lim _{h \rightarrow+\infty} I I_{h}=0
$$

By (5.18) and (5.19) we have

$$
\lim _{h \rightarrow+\infty} \frac{1}{8 \pi C} \int_{\Omega \backslash\left\{\left|\nabla w_{h}\right|=0\right\}}\left(\frac{1}{\beta_{\varepsilon_{h}}}+\beta_{\varepsilon_{h}}\left(\operatorname{div}\left(\frac{\nabla w_{h}}{\left|\nabla w_{h}\right|}\right)\right)^{2}\right)\left(\varepsilon_{h}\left|\nabla w_{\varepsilon_{h}}\right|^{2}+\frac{1}{\varepsilon_{h}} W\left(w_{\varepsilon_{h}}\right)\right) \mathrm{d} x=\mathcal{H}^{0}\left(P_{\nabla u}\right) .
$$

Now let us examine the terms involving $u_{h}$. As in the proof of Theorem 4.3 we have

$$
\limsup _{h \rightarrow+\infty} \int_{\Omega}\left|\nabla u_{h}-U_{0}\right|^{p} \mathrm{~d} x \leq \int_{\Omega}\left|\nabla u-U_{0}\right|^{p} \mathrm{~d} x
$$

Furthermore, by taking into account that $w_{h}(x)=1$ if $\delta_{D_{h}}(x) \geq 2 \varepsilon_{h}\left|\log \left(\varepsilon_{h}\right)\right|$ and $w_{h}(x)=0$ if $\delta_{D_{h}}(x)<$ $-2 \varepsilon_{h}\left|\log \left(\varepsilon_{h}\right)\right|$, by choosing $\rho_{h}$ small enough, we obtain

$$
\limsup _{h \rightarrow+\infty} \int_{\Omega} w_{h}^{2}\left|\Delta u_{h}\right|^{2} \mathrm{~d} x=\lim _{h \rightarrow+\infty} \int_{\Omega \backslash D_{h}^{0}}|\Delta u|^{2} \mathrm{~d} x=\int_{\Omega \backslash P_{\nabla u}}|\Delta u|^{2} \mathrm{~d} x=\int_{\Omega}|\Delta u|^{2} \mathrm{~d} x,
$$

where $D_{h}^{0}=\left\{x \in \Omega: \delta_{D_{h}}(x)<-2 \varepsilon_{h}\left|\log \left(\varepsilon_{h}\right)\right|\right\}$. Finally from the definition of $w_{h}$, it follows that $w_{h}=1$ outside the set $D_{2} \log \varepsilon_{h}:=\bigcup_{i=1}^{n} B_{2} \log \varepsilon_{h}\left(x_{i}\right)$ and hence thanks to (5.3) and (5.4)

$$
\limsup _{h \rightarrow+\infty} \frac{1}{\gamma_{h}} \int_{\Omega}\left(1-w_{h}\right)^{2} \mathrm{~d} x \leq \lim _{h \rightarrow+\infty} \mathcal{L}\left(D_{2} \log \varepsilon_{h}\right) \frac{1}{\gamma_{h}}=0 .
$$

The thesis follows by collecting (5.20), (5.21), (5.22) and (5.23). 


\subsection{Variational property}

Also in this case we obtain, as a direct consequence of Theorems 5.1, 5.2 and 5.3, the corresponding variational property. The proof is as in Theorem 4.4.

Theorem 5.4. Let $\mathcal{G}_{\varepsilon}$ and $\mathcal{F}$ be given respectively by (5.2) and (4.3). If $\left\{\varepsilon_{h}\right\}$ is a sequence of positive numbers converging to zero and $\left\{\left(u_{h}, w_{h}\right)\right\} \subset S(\Omega)$ such that

$$
\lim _{h \rightarrow+\infty}\left(\mathcal{G}_{\varepsilon_{h}}\left(u_{\varepsilon_{h}}, w_{\varepsilon_{h}}\right)-\inf _{S(\Omega)} \mathcal{G}_{\varepsilon_{h}}(u, w)\right)=0,
$$

then there exist a subsequence $\left\{\left(u_{h_{k}}, w_{h_{k}}\right)\right\} \subset S(\Omega)$ and a minimizer $\bar{u}$ of $\mathcal{F}(u)$, with $\bar{u} \in \Delta \mathcal{A} \mathcal{M}^{p, 2}(\Omega)$, such that $\left\{\left(u_{h_{k}}, w_{h_{k}}\right)\right\}_{k} \mathrm{H}$-converges to $\bar{u}$.

\section{De Giorgi's Conjecture}

One of the objectives of De Giorgi was to find a variational approximation of a curvature depending functional of the type:

$$
F^{2}(D)=\int_{\partial D}\left(1+\kappa^{2}\right) \mathrm{d} \mathcal{H}^{1}
$$

where $D$ is a regular set and $\kappa$ is a curvature of its boundary $\partial D$.

Since $\partial D$ can be represented as the discontinuity set of the function $w_{0}=1-\chi_{D}$, by Modica-Mortola's Theorem it follows that there is a sequence of non constant local minimizers such that $w_{\varepsilon} \rightarrow w_{0}$ with respect to the $L^{1}$-convergence and

$$
\lim _{\varepsilon \rightarrow 0} F_{\varepsilon}^{1}\left(w_{\varepsilon}\right):=C \mathcal{H}^{1}(\partial D)
$$

with $F_{\varepsilon}^{1}(w)=\int_{\Omega} \varepsilon|\nabla w|^{2}+\frac{1}{\varepsilon} W(w) \mathrm{d} x$ and $C=\int_{0}^{1} \sqrt{W(t)} \mathrm{d} t$.

Furthermore looking at the Euler-Lagrange equation associated to a contour length term, yields a contour curvature term $\kappa$, while the Euler-Lagrange equations for the functional $F_{\varepsilon}^{1}(w)$ contains a term $2 \varepsilon \Delta w-\frac{W^{\prime}(w)}{\varepsilon}$.

Then De Giorgi suggested to approximate by $\Gamma$-convergence the functional $F^{2}$ by adding to the ModicaMortola approximating functionals the following term

$$
F_{\varepsilon}^{2}(w)=\int_{\Omega}\left(2 \varepsilon \Delta w-\frac{W^{\prime}(w)}{\varepsilon}\right)^{2} \mathrm{~d} x .
$$

In [5] Bellettini and Paolini have proven the lim sup inequality, while the validity of the lim inf inequality for a modified version of the original conjecture has been proven by Röger and Shätzle (see [23]).

Inspired by the De Giorgi's conjecture (see [15] for the original statement) it appears natural to investigate, in the spirit of [9], the possibility of approximating the functional $\mathcal{F}$ by means of a sequence $\mathcal{F}_{\varepsilon}$ much more convenient from a numerical point view (see [19]):

$$
\begin{aligned}
\mathcal{F}_{\varepsilon}(u, w)= & \int_{\Omega} w^{2}|\Delta u|^{2} \mathrm{~d} x+\frac{1}{8 \pi C}\left(\frac{\beta_{\varepsilon}}{2 \varepsilon} \int_{\Omega}\left(2 \varepsilon \Delta w-\frac{W^{\prime}(w)}{\varepsilon}\right)^{2} \mathrm{~d} x+\frac{1}{\beta_{\varepsilon}} \int_{\Omega}\left(\varepsilon|\nabla w|^{2}+\frac{1}{\varepsilon} W(w)\right) \mathrm{d} x\right) \\
& +\int_{\Omega}\left|\nabla u-U_{0}\right|^{p} \mathrm{~d} x+\int_{\Omega} \frac{1}{\gamma_{\varepsilon}}\left(1-w^{2}\right) \mathrm{d} x .
\end{aligned}
$$

The presence of the term $\frac{1}{2 \varepsilon}$ will be clear in the proof. By the way we are able to prove only the $\Gamma$-limsup inequality. 
Theorem 6.1. Let $\left\{\varepsilon_{h}\right\}$ be a sequence of positive numbers converging to zero. For every $u \in \Delta \mathcal{A M}^{p, 2}(\Omega)$, there exists a sequence $\left\{\left(u_{h}, w_{h}\right)\right\}_{h} \subset S(\Omega)$ H-converging to $u$ such that

$$
\limsup _{h \rightarrow+\infty} \mathcal{F}_{\varepsilon_{h}}\left(u_{h}, w_{h}\right) \leq \mathcal{F}(u)
$$

Proof. Let $\left\{\left(u_{h}, w_{h}\right)\right\}_{h}$ be the optimal sequence of Theorem 5.3. It is not difficult to see that for every $x \in D_{h}^{1}$ the function $\delta_{h}(x)$ is regular and using the definition of $w_{h}$ and taking into account that $\eta^{\prime}=\sqrt{W(\eta)}$ the following identity holds

$$
2 \varepsilon_{h} \Delta w_{h}-\frac{W^{\prime}\left(w_{h}\right)}{\varepsilon_{h}}=2 \varepsilon_{h} \eta_{h}^{\prime} \Delta \delta_{D_{h}}(x)+2 \varepsilon \eta_{h}^{\prime \prime}-\frac{W^{\prime}\left(w_{h}\right)}{\varepsilon_{h}}=2 \varepsilon_{h} \eta_{h}^{\prime}\left(\delta_{D_{h}}(x)\right) .
$$

For $h$ large enough we also have $\Delta \delta_{D_{h}}(x)=\kappa\left(\left\{\delta_{D_{h}}(x)=t\right\}\right)$, for $t \in(0,1)$. Besides on $D_{h}^{1}$ we have $w_{h}(x)=$ $\eta\left(\frac{\delta_{D_{h}}(x)}{\varepsilon_{h}}\right)$ and hence the level set $\left\{\delta_{D_{h}}(x)=t\right\}$ corresponds to the level set $\left\{w_{h}(x)=\eta\left(\frac{t^{\prime}}{\varepsilon_{h}}\right)\right\}$ with $0 \leq \eta \leq 1$, so that we infer

$$
\kappa\left(\left\{\delta_{D_{h}}(x)=t\right\}\right)=\operatorname{div}\left(\frac{\nabla w_{h}}{\left|\nabla w_{h}\right|}\right) .
$$

By proceeding as in the proof of Theorem 5.3 and taking into account the equality $2 \varepsilon_{h}\left|\eta_{h}^{\prime}\left(\delta_{D_{h}}(x)\right)\right|=2 \varepsilon_{h}\left|\nabla w_{h}\right|$ we have

$$
\begin{aligned}
I_{h} & =\int_{D_{h}^{1}} \frac{\beta_{\varepsilon_{h}}}{2 \varepsilon_{h}}\left(2 \varepsilon_{h} \Delta w_{h}-\frac{W^{\prime}\left(w_{h}\right.}{\varepsilon_{h}}\right)^{2}+\frac{1}{\beta_{\varepsilon_{h}}}\left(\varepsilon_{h}\left|\nabla w_{h}\right|^{2}+\frac{1}{\varepsilon_{h}} W\left(w_{h}\right)\right) \mathrm{d} x \\
& =2 \int_{D_{h}^{1}}\left(\beta_{\varepsilon} \operatorname{div}\left(\frac{\nabla w_{h}}{\left|\nabla w_{h}\right|}\right)^{2}+\frac{1}{\beta_{\varepsilon_{h}}}\right) \sqrt{W\left(w_{h}\right)}\left|\nabla w_{h}\right| \mathrm{d} x .
\end{aligned}
$$

Then as in the proof of Theorem 5.3 we conclude

$$
\lim _{h \rightarrow+\infty} I_{h}=8 \pi \mathcal{H}^{0}\left(P_{\nabla u}\right) \int_{0}^{1} \sqrt{W(t)} \mathrm{d} t
$$

By the same calculation on $D_{h}^{2}$ one can check that the integral over $D_{h}^{2}$ vanishes as in the proof of Theorem 5.3.

The other terms can be estimated exactly as in the proof of Theorem 5.3 and therefore the thesis is achieved.

Acknowledgements. We warmly thank anonymous referees for their careful reading of the paper and numerous remarks. Their advice have permitted to significantly improve our work.

\section{REFERENCES}

[1] L. Ambrosio, N. Fusco and D. Pallara, Functions of bounded variation and free discontinuity problems. Oxford University Press, Oxford (2000).

[2] G. Anzellotti, Pairings between measures and bounded functions and compensated compactness. Ann. Mat. Pura Appl. 135 (1983) 293-318.

[3] G. Aubert, J. Aujol and L. Blanc-Feraud, Detecting codimension - Two objects in an image with Ginzburg-Landau models. Int. J. Comput. Vis. 65 (2005) 29-42.

[4] G. Bellettini, Variational approximation of functionals with curvatures and related properties. J. Conv. Anal. 4 (1997) 91-108.

[5] G. Bellettini and M. Paolini, Approssimazione variazionale di funzionali con curvatura. Seminario di Analisi Matematica, Dipartimento di Matematica dell'Università di Bologna (1993).

[6] F. Bethuel, H. Brezis and F. Hélein, Ginzburg-Landau Vortices. Birkäuser, Boston (1994). 
[7] A. Braides, Г-convergence for beginners. Oxford University Press, New York (2000).

[8] A. Braides and A. Malchiodi, Curvature theory of boundary phases: the two dimensional case. Interfaces Free Bound. 4 (2002) 345-370.

[9] A. Braides and R. March, Approximation by $\Gamma$-convergence of a curvature-depending functional in Visual Reconstruction. Comm. Pure Appl. Math. 59 (2006) 71-121.

[10] A. Chambolle and F. Doveri, Continuity of Neumann linear elliptic problems on varying two-dimensionals bounded open sets. Comm. Partial Diff. Eq. 22 (1997) 811-840.

[11] G.Q. Chen and H. Fried, Divergence-measure fields and conservation laws. Arch. Rational Mech. Anal. 147 (1999) 35-51.

[12] G.Q. Chen and H. Fried, On the theory of divergence-measure fields and its applications. Bol. Soc. Bras. Math. 32 (2001) $1-33$.

[13] G. Dal Maso, Introduction to Г-convergence. Birkhäuser, Boston (1993).

[14] G. Dal Maso, F. Murat, L. Orsina and A. Prignet, Renormalized solutions of elliptic equations with general measure data. Ann. Scuola Norm. Sup. Pisa Cl. Sci. 28 (1999) 741-808.

[15] E. De Giorgi, Some remarks on $\Gamma$-convergence and least square methods, in Composite Media and Homogenization Theory, G. Dal Maso and G.F. Dell'Antonio Eds., Birkhäuser, Boston (1991) 135-142.

[16] E. De Giorgi and T. Franzoni, Su un tipo di convergenza variazionale. Atti Accad. Naz. Lincei Rend. Cl. Sci. Mat. Natur. 58 (1975) 842-850.

[17] E. De Giorgi and T. Franzoni, Su un tipo di convergenza variazionale. Rend. Sem. Mat. Brescia 3 (1979) 63-101.

[18] L.C. Evans and R.F. Gariepy, Measure Theory and Fine Properties of Functions. CRC Press (1992).

[19] D. Graziani, L. Blanc-Feraud and G. Aubert, A formal $\Gamma$-convergence approach for the detection of points in 2-D images. SIAM J. Imaging Sci. (to appear).

[20] J. Heinonen, T. Kilpeläinen and O. Martio, Nonlinear Potential Theory of Degenerate Elliptic Equations. Oxford University Press, Oxford (1993).

[21] L. Modica, The gradient theory of phase transitions and the minimal interface criterion. Arch. Rational Mech. Anal. 98 (1987) $123-142$.

[22] L. Modica and S. Mortola, Un esempio di Г-convergenza. Boll. Un. Mat. Ital. 14-B (1977) 285-299.

[23] M. Röger and R. Shätzle, On a modified conjecture of De Giorgi. Math. Zeitschrift 254 (2006) 675-714.

[24] G. Stampacchia, Le problème de Dirichlet pour les équations elliptiques du second ordre à coefficients discontinus. Ann. Inst. Fourier (Grenoble) 15 (1965) 180-258.

[25] W. Ziemer, Weakly Differentiable Functions. Springer-Verlag, New York (1989). 